TRANSACTIONS OF THE

AMERICAN MATHEMATICAL SOCIETY

Volume 364, Number 9, September 2012, Pages 4629-4662

S 0002-9947(2012)05429-8

Article electronically published on April 12, 2012

\title{
ON THE SOLVABILITY OF PLANAR COMPLEX LINEAR VECTOR FIELDS
}

\author{
FRANÇOIS TREVES
}

\begin{abstract}
The article discusses the local solvability, or lack thereof, of vector fields whose coefficients are complex-valued linear functions in $\mathbb{R}^{2}$ (here called complex linear). It is proved that such a vector field is locally solvable in $\mathbb{R}^{2}$ if and only if it is locally solvable and does not have compact orbits in the complement of its critical set or, equivalently, its Meziani number is different from zero.
\end{abstract}

\section{Contents}

1. Introduction 4630

1.1. Statement of result 4630

1.2. Necessity of the conditions in Theorem 1, Part (B) 4632

2. Classification and invariants 4634

2.1. Classification of vector fields with linear coefficients 4634

2.2. Square root of $A(\theta) \quad 4637$

2.3. The invariant $K(\pi)$

2.4. Hypoellipticity, ellipticity and local solvability conditions
in $\mathbb{R}^{2} \backslash\{0\}$

2.5. Necessity of the conditions in Theorem 3

3. Solvability in the classes (NO-E) 4644

3.1. Cases (NO-E). Preparatory lemmas 4645

3.2. Vector field elliptic in $\mathbb{R}^{2} \backslash\{0\}$. Case $\mu(L) \in \mathbb{R}$

3.3. Vector field elliptic in $\mathbb{R}^{2} \backslash\{0\}$. Case $\mu(L) \notin \mathbb{R}$

3.4. Vector field hypoelliptic but not elliptic in $\mathbb{R}^{2} \backslash\{0\}$.
Case $\mu(L) \in \mathbb{R}$

3.5. Vector field hypoelliptic but not elliptic in $\mathbb{R}^{2} \backslash\{0\}$.

Case $\mu(L) \notin \mathbb{R}$

3.6. End of the proof of sufficiency in the class (NO-E)

4. Solvability in the class (ON-E) 4655

4.1. Preparatory reductions 4655

5. A microlocal remark 4660

5.1. Property (NO-E) in phase space 4660

5.2. Comparing Theorem 12 to a classical theorem of Hörmander 4661

References 4661

Received by the editors November 29, 2009 and, in revised form, July 22, 2010.

2010 Mathematics Subject Classification. Primary 35A01, 35F05; Secondary 35D30, 35H10.

(C)2012 American Mathematical Society

Reverts to public domain 28 years from publication 


\section{INTRODUCTION}

1.1. Statement of result. The present work describes the solvability, or lack thereof, of planar vector fields $L$ with complex linear coefficients,

$$
L=(a x+b y) \partial_{x}+(c x+d y) \partial_{y} .
$$

We recall some standard terminology and notation. A critical point $\left(x^{\circ}, y^{\circ}\right)$ of $L$ is a point where $L=0$ : the vector $\left(\begin{array}{l}x^{\circ} \\ y^{\circ}\end{array}\right)$ belongs to the null space of the complex $2 \times 2$ matrix $\mathfrak{m}(L)=\left(\begin{array}{ll}a & b \\ c & d\end{array}\right)$. We exclude the case $a=b=c=d=0$ : the critical points of $L$ either form a whole straight line through the origin or else reduce to $\{0\}$. The trace $\operatorname{tr} \mathfrak{m}(L)=a+d$ is also the divergence of $L, \operatorname{div} L$.

We denote by $\mathcal{C}_{\mathrm{c}}^{\infty}(\Omega)$ the algebra of test functions in the open set $\Omega \subset \mathbb{R}^{2}$, the complex-valued $\mathcal{C}^{\infty}$ functions in $\mathbb{R}^{2}$ that vanish identically in the complement of some compact subset of $\Omega ; \mathcal{D}^{\prime}(\Omega)$ stands for the dual of $\mathcal{C}_{\mathrm{c}}^{\infty}(\Omega)$, i.e., the space of distributions in $\Omega$.

We say that $L$ is globally solvable, or solvable, for short, in an open subset $\Omega$ of $\mathbb{R}^{2}$ if the following holds:

$\forall f \in \mathcal{C}_{\mathrm{c}}^{\infty}(\Omega), \exists u \in \mathcal{D}^{\prime}(\Omega)$ verifying $L u=f$ in $\Omega$.

The vector field $L$ is said to be semiglobally solvable in $\mathbb{R}^{2}$ if it is globally solvable in every bounded open subset of $\mathbb{R}^{2} ; L$ is said to be locally solvable at a point of $\mathbb{R}^{2}$ if $L$ is solvable in some (open) neighborhood of the point. Furthermore, $L$ is said to be locally solvable in a set $S \subset \mathbb{R}^{2}$ if it is locally solvable at every point of $S$. The vector fields (1.1) being invariant under dilations $(x, y) \longrightarrow(\lambda x, \lambda y), \lambda>0$, local and semiglobal solvability in $\mathbb{R}^{2}$ are equivalent.

The central result of this article can be stated as follows:

Theorem 1. (A): If the critical points of the vector field (1.1) form a straight line, then $L$ is globally solvable in $\mathbb{R}^{2}$.

(B): If the origin is the only critical point of $L$, then, for $L$ to be locally solvable in $\mathbb{R}^{2}$, it is necessary and sufficient that the following two conditions be satisfied:

(i): $L$ is locally solvable in $\mathbb{R}^{2} \backslash\{0\}$;

(ii): L has no compact orbit in $\mathbb{R}^{2} \backslash\{0\}$.

By an orbit of $L$ in $\mathbb{R}^{2} \backslash\{0\}$ we mean a one-dimensional connected, immersed submanifold of class $\mathcal{C}^{\omega}$ of $\mathbb{R}^{2} \backslash\{0\}$ to which $L$ is tangent at every one of its points and which is maximal for these properties. Actually, owing to the very special nature of the vector fields (1.1), the preceding statement will be shown to remain valid if we weaken Condition (ii) to

(ii'): L has no orbit in $\mathbb{R}^{2} \backslash\{0\}$ whose closure in $\mathbb{R}^{2}$ is a compact subset of $\mathbb{R}^{2} \backslash\{0\}$.

Orbits whose closure is compact are commonly said to be "trapped".

Remark 1. Suppose that $\zeta L$ is a real vector field for some $\zeta \in \mathbb{C} \backslash\{0\}$. To say that the orbits of $\zeta L$ in $\mathbb{R}^{2} \backslash\{0\}$ are trapped is equivalent to saying that the oneparameter subgroup $\mathbb{R} \ni t \longrightarrow \exp (t \zeta \mathfrak{m}(L))$ has a compact closure in $\mathbf{G L}(2, \mathbb{C})$.

The proof of Part (A) is very simple: After a rotation we may assume that $(0,1)$ is a critical point of $L$, implying $L=x\left(a \partial_{x}+c \partial_{y}\right)$. Every distribution $f$ in $\mathbb{R}^{2}$ can be divided by an analytic function (here $x$ ) to yield a distribution in $\mathbb{R}^{2}$ 
(Lojasiewiccz, 1965) and the equation

$$
a \partial_{x} u+c \partial_{y} u=x^{-1} f
$$

has a solution $u \in \mathcal{D}^{\prime}\left(\mathbb{R}^{2}\right)$ (Hörmander, 1959 or Hörmander, 1969 Corollary 3.6.2).

In proving Part (B), Theorem 1, we shall prove a version that is more easily stated in the negative:

Theorem 2. Assume that the vector field (1.1) is locally solvable in $\mathbb{R}^{2} \backslash\{0\}$. For $L$ not to be locally solvable at the origin it is necessary and sufficient that there be a complex number $\zeta \neq 0$ such that $\zeta L$ is a real vector field whose orbits in $\mathbb{R}^{2} \backslash\{0\}$ are ellipses centered at the origin or, equivalently, which can be transformed into $\partial_{\theta}=x \partial_{y}-y \partial_{x}$ by a linear change of the coordinates in $\mathbb{R}^{2}$.

An earlier, unpublished, version of Theorem 1 characterized the local solvability of $L$ in terms of a complex number (more accurately, an element of the Riemann sphere) $\mu(L)$ invariantly associated to $L$, which we call the Meziani invariant of $L$ and which was first introduced in the study Meziani, 2001 of a planar complex vector field (without critical points) elliptic off a simple closed curve $\mathfrak{c}$ and uniformly tangent to it (to first order).

We introduce the Meziani invariant of $L, \mu(L)$, without further ado. In polar coordinates,

$$
L=\frac{1}{2}\left((a+d)+A^{\prime}(\theta)\right) r \partial_{r}-A(\theta) \partial_{\theta}
$$

where

$$
\begin{aligned}
A(\theta) & =b \sin ^{2} \theta+(a-d) \cos \theta \sin \theta-c \cos ^{2} \theta \\
& =\frac{1}{2}(b-c)-\frac{1}{2}(b+c) \cos 2 \theta+\frac{1}{2}(a-d) \sin 2 \theta
\end{aligned}
$$

is periodic of period $\pi$.

Definition 1. If $A\left(\theta^{\circ}\right)=0$ for some $\theta^{\circ} \in \mathbb{R}$ we posit $\mu(L)=\infty$. If $A(\theta) \neq 0$ for all $\theta \in \mathbb{R}$,

$$
\mu(L)=\frac{1}{2 i \pi} \int_{0}^{\pi} \frac{A^{\prime}(\theta)+\operatorname{div} L}{A(\theta)} d \theta .
$$

In Meziani, 2001 it is shown, under the hypothesis that $\mu(L)$ is not a real number, how to put the vector field in normal form in a tubular neighborhood of $\mathfrak{c}$, by means of diffeomorphisms of finite regularity. We shall use Meziani's result in the case where $L$ is elliptic in $\mathbb{R}^{2} \backslash\{0\}$. The situation studied in Meziani, 2001 was later completely clarified in Cordaro-Gong, 2004; in particular, the restriction $\mu(L) \notin \mathbb{R}$ was weakened to $\mu(L) \notin \mathbb{Q}$.

It will be shown that Theorem 1 can be restated as follows:

Theorem 3. For the vector field (1.1) to be locally solvable in $\mathbb{R}^{2}$ it is necessary and sufficient that $L$ be locally solvable in $\mathbb{R}^{2} \backslash\{0\}$ and that $\mu(L)$ not be equal to zero.

Note that, when the critical points of the vector field (1.1) form a straight line $z=t e^{i \theta^{\circ}}$, then $A\left(\theta^{\circ}\right)=0$ and $\mu(L)=\infty$, which shows that the statement of Theorem 3 "covers" Part (A) of Theorem 1, We assume, in the remainder of the article, that the origin is the sole critical point of $L$. In passing, note that, the 
matrix $\mathfrak{m}(L)$ being complex, the property that 0 is the sole critical point of $L$ does not necessarily mean that $\operatorname{det} \mathfrak{m}(L) \neq 0$. Example: $L=(x+i y) \partial_{x}$.

Our proof of Theorems 1, 2 and 3] is based on a classification of the vector fields with linear coefficients into three classes (Subsection 2.1), defined respectively by properties called (IN-E) ("inside the ellipse"), (OUT-E) ("outside the ellipse") and (ON-E) ("on the ellipse"), each invariant under linear changes of the real variables in the plane (Theorem 5). The ellipse (possibly a straight-line segment) referred to here is the range of the map $[0,2 \pi) \ni \theta \longrightarrow 2 A\left(\frac{1}{2} \theta\right) \in \mathbb{C}$; "IN", "OUT" and "ON" refer to the location of the origin relative to said ellipse. The vector fields in the class (ON-E) are tangent to some straight line through the origin (possibly vanishing identically on it) and, in accordance with Definition 1, they are characterized by the property that $\mu(L)=\infty$. The other two classes, (IN-E) or (OUT-E), are distinguished by the vanishing or nonvanishing, respectively, of an invariant complex number $K(\pi)$ (Theorem 6). The Meziani invariant is related to this other invariant: $\mu(L)=K(\pi) \operatorname{div} L$ in the class (OUT-E); whereas $\mu(L)=$ \pm 1 in the class (IN-E). Incidentally, this reveals that, according to Theorem 3 , the nonsolvable vector fields with linear coefficients are those that satisfy (OUT-E), and thus satisfy $K(\pi) \neq 0$, and are divergence free (recall that the divergence of a smooth vector field at a critical point $\wp$ is invariant under diffeomorphisms that preserve $\wp)$.

Before embarking on a description of the classification and its use we will establish directly the necessity of the conditions in Theorem 1 Part (B). We shall deduce the necessity of Condition (ii) from a result of Müller, 1992. In Subsection 2.5 a simple proof of the same result will be derived within the framework of the classification.

The vector fields (1.1) make up the complement of the origin in a complex Lie algebra $\mathfrak{g}_{\text {lin }}$ in which the Lie bracket is the commutation bracket of differential operators. It is directly checked that if $L_{1}$ and $L_{2}$ are two elements of $\mathfrak{g}_{\text {lin }}$, then $\mathfrak{m}\left(\left[L_{1}, L_{2}\right]\right)=-\left[\mathfrak{m}\left(L_{1}\right), \mathfrak{m}\left(L_{2}\right)\right]$. The map $L \longrightarrow \mathfrak{m}(L)$ extended to $L=0$ is a Lie algebra isomorphism of $\mathfrak{g}_{\text {lin }}$ onto the Lie algebra of complex $2 \times 2$ matrices $\mathbf{M}_{2}(\mathbb{C})$ provided we flip the sign of the Lie bracket in one of these two Lie algebras. If $\Gamma=\left(\begin{array}{ll}\alpha & \beta \\ \gamma & \delta\end{array}\right) \in \mathbf{G L}(2, \mathbb{R})$ the change of variables $(x, y) \longrightarrow(\alpha x+\beta y, \gamma x+\delta y)$ transforms $\mathfrak{m}(L)$ into $\Gamma^{-1} \mathfrak{m}(L) \Gamma$ and thus $L$ into $\underset{\mathfrak{m}}{-1}\left(\Gamma^{-1} \mathfrak{m}(L) \Gamma\right)$.

1.2. Necessity of the conditions in Theorem 1, Part (B). The necessity of Property (i) in Part (B) of Theorem 1 is evident. In order to prove the necessity of Property (ii) we need the following.

Proposition 1. Assume that 0 is the only critical point of the vector field (1.5). If Property (ii) in Part (B) of Theorem 1 is not true, then every one of the following properties is true:

(1) there is a complex number $\zeta \neq 0$ such that the vector field $\zeta L$ is real;

(2) $\operatorname{div} L=0$;

(3) there is a linear change of coordinates in the plane that transforms the orbits of $L$ into the circles centered at the origin;

(4) there is a complex number $\zeta \neq 0$ such that the eigenvalues of $\mathfrak{m}(\zeta L)$ are equal to $\pm i$. 
Conversely, the validity of these properties implies the negation of Property (ii) in Theorem 1, Part (B).

Remark 2. If $e^{i \theta^{\circ}}$ is not a critical point of $L$ and if $A\left(\theta^{\circ}\right)=0$, then the two rays $\pm r e^{i \theta^{\circ}}$ are orbits of $L$ in $\mathbb{R}^{2} \backslash\{0\}$.

Proof. Suppose there is a compact orbit $\mathfrak{c}$ of $L$ in $\mathbb{R}^{2} \backslash\{0\}: L$ is a complex multiple of the unit tangent vector to $\mathfrak{c}$ at every point of $\mathfrak{c}$. We cannot have $A(\theta)=0$ for any point $r e^{i \theta} \in \mathfrak{c}$ (by Remark 2) and thus, by (1.2), the radial vector $\partial_{r}$ is nowhere tangent to $\mathfrak{c}$. The radial span of $\mathfrak{c}$ is a cone relatively closed in $\mathbb{R}^{2} \backslash\{0\}$, with interior $\Omega \neq \varnothing$. If a ray $r e^{i \theta^{\circ}}(r>0)$ were part of the boundary of $\Omega$, such a ray would be tangent to $\mathfrak{c}$ at some point $r^{\circ} e^{i \theta^{\circ}}$, an impossibility (again by Remark 2). We conclude that $\Omega=\mathbb{R}^{2} \backslash\{0\}$ and that $A(\theta) \neq 0$ for all $\theta \in \mathbb{R}$. As a consequence, $L$ is the complex multiple of a real vector field at every point of $\mathbb{R}^{2}$, which is the same as saying that

$$
\frac{1}{2 i} L \wedge \bar{L}=\operatorname{Im}((a x+b y)(\bar{c} x+\bar{d} y)) \partial_{x} \wedge \partial_{y}
$$

vanishes identically in $\mathbb{R}^{2}$ or, equivalently, that

$$
\operatorname{Im}(a \bar{c})=\operatorname{Im}(b \bar{d})=\operatorname{Im}(a \bar{d}+b \bar{c})=0 .
$$

First suppose $a+d=0$; in this case (1.6) reduces to the statement that $a \bar{c}, a \bar{b}$ and $b \bar{c}$ are real, implying that $\zeta L$ is real for some $\zeta \in \mathbb{C}, \zeta \neq 0$. Now suppose $a+d \neq 0$ and, say, $d \neq 0$. After division by $d$ we may assume $d=1$. From (1.6) we derive, first, that $b \in \mathbb{R}$. Keep in mind that $A(\theta) \neq 0$ for all $\theta$ demands $b c \neq 0$; 1.6) implies $a=s c$ for some $s \in \mathbb{R}$ and, then, $(b-s) \operatorname{Im} c=0$. If $b \neq s$ we conclude, here also, that $L$ is real. Otherwise we get $a=b c$. But, in the latter case,

$$
\begin{aligned}
A(\theta) & =b \sin ^{2} \theta+(b c-1) \cos \theta \sin \theta-c \cos ^{2} \theta \\
& =(\sin \theta+c \cos \theta)(-\cos \theta+b \sin \theta)
\end{aligned}
$$

vanishes when $\cot \theta=b$, which contradicts our findings above. This completes the proof of Property \#1. Now assume $L$ is real and $A(\theta) \neq 0$ for all $\theta \in \mathbb{R}$. We derive from (1.2) that the orbits of $L$ are defined by the equation

$$
r=\rho \exp \left(-\frac{1}{2} \int_{0}^{\theta} \frac{a+d+A^{\prime}(\tau)}{A(\tau)} d \tau\right)
$$

where $\rho \in \mathbb{R}$ is a constant. If we had

$$
\kappa=\int_{0}^{2 \pi} \frac{a+d+A^{\prime}(\tau)}{A(\tau)} d \tau=(a+d) \int_{0}^{2 \pi} \frac{d \tau}{A(\tau)}
$$

not equal to zero, say $\kappa>0$, then the curve defined by (1.7) would spiral to 0 when $\theta \longrightarrow+\infty$ and to $\infty$ when $\theta \longrightarrow-\infty$, contradicting the compactness of $\mathfrak{c}$. Since the real function $A$ is nowhere zero, $\kappa=0 \Longleftrightarrow a+d=0$; this proves Property \#2. In turn, the latter implies that (1.7) reduces to

$$
r \sqrt{|A(\theta)|}=\rho,
$$

which is easily seen to be the equation of an ellipse centered at the origin. There is a linear change of variables $(x, y) \longrightarrow(\alpha x+\beta y, \gamma x+\delta y)$, with $\alpha \delta-\beta \gamma \neq 0$, that transforms the ellipse (1.8) into the circle $r=\rho$ and $L$ into $\lambda\left(x \partial_{y}-y \partial_{x}\right)$, $\lambda \in \mathbb{R} \backslash\{0\}$. This proves Properties \#3 and \#4 in Proposition 1 The converse assertion is self-evident. 
We state here the 2D version of the main result of [Treves, 2009.

Theorem 4. Let $L$ be the vector field (1.1) and assume that 0 is its only critical point. If there is a complex number $\zeta \neq 0$ such that $\zeta L$ is real, then the following properties are equivalent:

(a): $L$ is not locally solvable at the origin.

(b): The closure of every orbit of $L$ in $\mathbb{R}^{2} \backslash\{0\}$ is a compact subset of $\mathbb{R}^{2} \backslash\{0\}$.

(c): There is a linear change of coordinates in the plane that transforms the orbits of $L$ into the circles centered at the origin.

(d): The eigenvalues of $\mathfrak{m}(\zeta L)$ are equal to $\pm i \eta, 0 \neq \eta \in \mathbb{R}$.

The equivalence of (a) and (d) is a very particular case of Theorem 1.2, Müller, 1992.

Combining Proposition 1 and Theorem 4 proves that Condition (ii) in Part (B) of Theorem 1 is necessary. It also proves the sufficiency of the condition in Theorem 2

\section{Classification and invariants}

2.1. Classification of vector fields with linear coefficients. Returning to the polar coordinates expressions (1.2)-(1.3) we use the simpler notation

$$
A(\theta)=\frac{1}{2}(\alpha \sin 2 \theta-\beta \cos 2 \theta+\gamma),
$$

where

$$
\alpha=a-d, \beta=b+c, \gamma=b-c .
$$

As $\theta$ ranges over the segment $[0,2 \pi]$ the complex number $2 A\left(\frac{1}{2} \theta\right)$ describes the ellipse $\mathfrak{E}(A)$ centered at $\gamma$, defined by the parametric equations (in complex notation $z=x+i y)$ :

$$
z-\gamma=\alpha \sin \theta-\beta \cos \theta .
$$

If $\operatorname{Im}(\alpha \bar{\beta}) \neq 0$ we can define $\mathfrak{E}(A)$ by the implicit equation

$$
(\operatorname{Im}(\bar{\alpha}(z-\gamma)))^{2}+(\operatorname{Im}(\bar{\beta}(z-\gamma)))^{2}=(\operatorname{Im}(\alpha \bar{\beta}))^{2} .
$$

If $\operatorname{Im}(\alpha \bar{\beta})=0$ we can rewrite (2.3) as

$$
z-\gamma=\zeta \sin (\theta-\phi)
$$

with $\phi \in[0,2 \pi)$ and $\zeta=t \alpha$ or $\zeta=t \beta$ for some $t \in \mathbb{R}$. When $\mathfrak{E}(A)$ is defined by (2.5) it is a segment. We have $\mathfrak{E}(A)=\{\gamma\}$ if and only if $\alpha=\beta=0$.

We subdivide the set of vector fields (1.1), $\mathfrak{g}_{\text {lin }}$, into the three classes defined by the following properties:

(IN-E): The origin lies inside the ellipse $\mathfrak{E}(A)$, meaning that

$$
(\operatorname{Im}(\bar{\alpha} \gamma))^{2}+(\operatorname{Im}(\bar{\beta} \gamma))^{2}<(\operatorname{Im}(\alpha \bar{\beta}))^{2} .
$$

(OUT-E): The origin lies outside the ellipse $\mathfrak{E}(A)$, meaning either that

$$
(\operatorname{Im}(\bar{\alpha} \gamma))^{2}+(\operatorname{Im}(\bar{\beta} \gamma))^{2}>(\operatorname{Im}(\alpha \bar{\beta}))^{2}
$$

when $\operatorname{Im}(\alpha \bar{\beta}) \neq 0$, or, when $\mathfrak{E}(A)$ is defined by (2.5), that $\gamma \neq t \zeta$ whatever $t \in[-1,1]$. 
(ON-E): The origin lies on the ellipse $\mathfrak{E}(A)$, meaning either that

$$
(\operatorname{Im}(\bar{\alpha} \gamma))^{2}+(\operatorname{Im}(\bar{\beta} \gamma))^{2}=(\operatorname{Im}(\alpha \bar{\beta}))^{2}
$$

when $\operatorname{Im}(\alpha \bar{\beta}) \neq 0$, or, when $\mathfrak{E}(A)$ is defined by (2.5), that $\gamma=t \zeta$ for some $t \in[-1,1]$.

To be precise: (IN-E) means that $\mathfrak{E}(A)$ is a true ellipse, not a segment, and that 0 belongs to the convex hull of $\mathfrak{E}(A)$ but not to its boundary; (OUT-E) means that 0 lies in the complement of the closed convex hull of $\mathfrak{E}(A) ;(\mathbf{O N}-\mathbf{E})$ means that $0 \in \mathfrak{E}(A)$, i.e., $A(\theta)=0$ for some $\theta \in \mathbb{R}$. It will be convenient to use one notation for the negation of (ON-E) or, which is the same, the conjunction of (IN-E) and (OUT-E):

(NO-E): $\forall \theta \in \mathbb{R}, A(\theta) \neq 0$.

Remark 3. It is evident that each one of the properties (IN-E), (OUT-E) and (ON-E) is invariant under complex conjugation $L \longrightarrow \bar{L}$ and multiplication $L \longrightarrow$ $\zeta L, 0 \neq \zeta \in \mathbb{C}$.

Taking (2.4) into account gets us

$$
\begin{gathered}
\operatorname{Im}(\beta \bar{\gamma})=\operatorname{Im}((b+c)(\bar{b}-\bar{c}))=-2 \operatorname{Im}(b \bar{c}), \\
(\operatorname{Im}(\alpha \bar{\beta}))^{2}-(\operatorname{Im}(\alpha \bar{\gamma}))^{2}=4 \operatorname{Im}(\alpha \bar{b}) \operatorname{Im}(\alpha \bar{c}) .
\end{gathered}
$$

We see that (2.6) is equivalent to

$$
(\operatorname{Im}(b \bar{c}))^{2}<(\operatorname{Im}((a-d) \bar{c}))(\operatorname{Im}((a-d) \bar{b})) ;
$$

(2.7) is equivalent to

$$
(\operatorname{Im}((a-d) \bar{c}))(\operatorname{Im}((a-d) \bar{b}))<(\operatorname{Im}(b \bar{c}))^{2},
$$

while (2.8) is equivalent to

$$
(\operatorname{Im}((a-d) \bar{c}))(\operatorname{Im}((a-d) \bar{b}))=(\operatorname{Im}(b \bar{c}))^{2} .
$$

We introduce the fundamental symplectic matrix

$$
\digamma=\left(\begin{array}{cc}
0 & -1 \\
1 & 0
\end{array}\right)
$$

Let $M^{*}=\left(\frac{\bar{a}}{b} \frac{\bar{c}}{d}\right)$ be the adjoint of the matrix $M=\left(\begin{array}{ll}a & b \\ c & d\end{array}\right)$. The spectral properties of the following matrix will determine the class of $L$ :

$$
\begin{aligned}
\mathfrak{T}(L) & =\frac{1}{2 i}\left[\mathfrak{m}(L), \digamma \mathfrak{m}(L)^{*} \digamma\right] \\
& =\operatorname{Im}\left(\begin{array}{cc}
b \bar{c} & (a-d) \bar{b} \\
-(a-d) \bar{c} & -b \bar{c}
\end{array}\right) .
\end{aligned}
$$

Theorem 5. Property (2.6) holds if and only if $\mathfrak{T}(L)$ has eigenvalues $\pm i \lambda, 0 \neq$ $\lambda \in \mathbb{R}$. Property (2.7) holds if and only if $\mathfrak{T}(L)$ has eigenvalues $\pm \lambda, 0 \neq \lambda \in \mathbb{R}$. Property (2.8) holds if and only if $\mathfrak{T}(L)^{2}=0$ but $\mathfrak{T}(L) \neq 0$. In all these cases $\mathfrak{E}(A)$ is a true ellipse; $\mathfrak{E}(A)$ is a compact segment of a straight line if and only if $\mathfrak{T}(L)=0$. Each one of these properties is invariant under linear changes of the variables $x, y$ in $\mathbb{R}^{2}$. 
Proof. A simple calculation shows that

$$
\operatorname{det}(\mathfrak{T}(L))=\operatorname{Im}((a-d) \bar{b}) \operatorname{Im}((a-d) \bar{c})-(\operatorname{Im}(b \bar{c}))^{2} .
$$

We derive immediately that (2.9) is equivalent to $\operatorname{det}(\mathfrak{T}(L))>0$; (2.10) is equivalent to $\operatorname{det}(\mathfrak{T}(L))<0$; (2.11) is equivalent to $\operatorname{det}(\mathfrak{T}(L))=0$. Since $\operatorname{tr} \mathfrak{T}(L)=0$ we reach the desired conclusions, namely that Property (2.9) holds if and only if $\mathfrak{T}(L)$ has eigenvalues $\pm i \lambda, 0 \neq \lambda \in \mathbb{R}$; Property (2.10) holds if and only if $\mathfrak{T}(L)$ has eigenvalues $\pm \lambda, 0 \neq \lambda \in \mathbb{R}$; Property (2.11) holds if and only if

$$
\mathfrak{T}(L)^{2}=0 .
$$

Now let $\Gamma \in \mathbf{G L}(2, \mathbb{R})$. We have $\Gamma \digamma \Gamma^{\top}=(\operatorname{det} \Gamma) \digamma$ whence

$$
\begin{aligned}
\Gamma^{-1} M \Gamma \digamma \Gamma^{\top} M^{*}\left(\Gamma^{-1}\right)^{\top} \digamma & =(\operatorname{det} \Gamma) \Gamma^{-1} M \digamma M^{*}\left(\Gamma^{-1}\right)^{\top} \digamma \Gamma^{-1} \Gamma \\
& =\Gamma^{-1} M \digamma M^{*} \digamma \Gamma
\end{aligned}
$$

and thus

$$
\left[\Gamma^{-1} M \Gamma, \digamma\left(\Gamma^{-1} M \Gamma\right)^{*} \digamma\right]=\Gamma^{-1} \mathfrak{T}(L) \Gamma .
$$

We have just seen that Property (2.7) holds if and only if the real matrix $\mathfrak{T}(L)$ has distinct real eigenvalues; it follows that it has real diagonalizers: thanks to (2.15) we see that (2.7) holds if and only if there is a linear change of the coordinates in $\mathbb{R}^{2}$ which results in the expression

$$
\mathfrak{T}(L)=\lambda\left(\begin{array}{cc}
1 & 0 \\
0 & -1
\end{array}\right)
$$

with $0 \neq \lambda \in \mathbb{R}$. After such a change of variables we have $\operatorname{Im}(b \bar{c})=\lambda \neq 0$ and

$$
\operatorname{Im}((a-d) \bar{b})=\operatorname{Im}((a-d) \bar{c})=0 .
$$

Equation (2.16) implies that $b^{-1}(a-d) \in \mathbb{R}, c^{-1}(a-d) \in \mathbb{R}$. If we had $a \neq d$ we would have $b^{-1} c \in \mathbb{R}$, a contradiction. We conclude that (2.7) is equivalent to the following property:

$(\bullet)$ : There is a linear change of the coordinates in $\mathbb{R}^{2}$ which yields the expression

$$
A(\theta)=\frac{1}{2}(b-c)-\frac{1}{2}(b+c) \cos 2 \theta, \operatorname{Im}(b \bar{c}) \neq 0 .
$$

If (2.17) holds, then $\mathfrak{E}(A)$ is an ellipse contained in $\mathbb{C} \backslash\{0\}$, implying (OUT-E). Finally suppose that (2.9) holds. There are two possibilities:

(1) there exists $\Gamma \in \mathbf{G L}(2, \mathbb{R})$ such that

$$
\Gamma^{-1} \mathfrak{T}(L) \Gamma=\left(\begin{array}{cc}
0 & 1 \\
0 & 0
\end{array}\right) ;
$$

(2) $\mathfrak{T}(L)=0$.

Case 1. In the coordinate system resulting from the linear transformation associated with $\Gamma$ we must have $\operatorname{Im}(b \bar{c})=\operatorname{Im}(\alpha \bar{c})=0$, implying $c=s b$ and $c=t \alpha$, $s, t \in \mathbb{R}$. But since we must also have $\operatorname{Im}(\alpha \bar{b})=1$, this is only possible if $c=0$. Thus (2.9) yields $A(\theta)=(\alpha \cos \theta+b \sin \theta) \sin \theta$ with $\operatorname{Im}(\alpha \bar{b}) \neq 0$. Here $\mathfrak{E}(A)$ is a "true" ellipse passing through the origin. 
Case 2. According to (2.13) we have $\operatorname{Im}(b \bar{c})=\operatorname{Im}(\alpha \bar{b})=\operatorname{Im}(\alpha \bar{c})=0$. If $|b|+|c| \neq$ $0,(2.9)$ demands $\alpha=a-d=0$ and we get (2.17) but this time with $\operatorname{Im}(b \bar{c})=0$, which means that $\mathfrak{E}(A)$ is the straight-line segment (possibly reduced to a single point) joining the points $b$ and $-c$. If $b=c=0$, we get $A(\theta)=\frac{1}{2} \alpha \sin 2 \theta$, which means that $\mathfrak{E}(A)$ is the straight-line segment (possibly reduced to a single point) joining the points $\pm \frac{1}{2} \alpha$. The invariance of all these properties under linear changes of variables follows directly from (2.15).

Corollary 1. Each one of the properties (IN-E), (OUT-E) and $(\boldsymbol{O N}-\boldsymbol{E})$ is invariant under linear changes of the variables $x, y$ in $\mathbb{R}^{2}$.

Proposition 2. For Property (IN-E) to hold it is necessary and sufficient that $\partial_{\theta}(\arg A(\theta)) \neq 0$ for every $\theta \in \mathbb{R}$.

Proof. Using the fact that $\partial_{\theta}(\arg A)=|A|^{-2} \operatorname{Im}\left(A^{\prime} \bar{A}\right)$ we deduce from (2.1):

$$
2 \operatorname{Im}\left(A(\theta) \bar{A}^{\prime}(\theta)\right)=\operatorname{Im}(\alpha \bar{\beta})-\operatorname{Im}(\alpha \bar{\gamma}) \cos 2 \theta-\operatorname{Im}(\beta \bar{\gamma}) \sin 2 \theta .
$$

In view of this and using $t=\tan \theta$ as a parameter we can rewrite the equation $\operatorname{Im}\left(A(\theta) \bar{A}^{\prime}(\theta)\right)=0$ as

$$
\operatorname{Im}(\alpha \bar{\beta})-\operatorname{Im}(\beta \bar{\gamma})-2 \operatorname{Im}(\alpha \bar{\gamma}) t+(\operatorname{Im}(\alpha \bar{\beta})+\operatorname{Im}(\beta \bar{\gamma})) t^{2}=0 .
$$

To say that this equation in $t$ has real solutions is equivalent to saying that

$$
(\operatorname{Im}(\bar{\alpha} \gamma))^{2}+(\operatorname{Im}(\bar{\beta} \gamma))^{2} \geq(\operatorname{Im}(\alpha \bar{\beta}))^{2},
$$

which is exactly the negation of (2.6). We conclude that Property (2.6) is equivalent to the fact that $\operatorname{Im}\left(A(\theta) \bar{A}^{\prime}(\theta)\right) \neq 0$ for every $\theta \in \mathbb{R}$.

Remark 4. The classification of vector fields $L$ completely ignores $\operatorname{div} L$ : it only involves the complex numbers $a-d, b, c$. It really only classifies the vector fields $L_{\circ}=L-\frac{1}{2}(\operatorname{div} L) r \partial_{r}$. Note that the matrices $\mathfrak{m}\left(L_{\circ}\right)$ form the Lie algebra of complex matrices whose trace vanishes, i.e., $\mathfrak{s l}(2, \mathbb{C})$, identical to the commutator subalgebra of $\mathbf{M}_{2}(\mathbb{C})$.

\subsection{Square root of $A(\theta)$.}

Lemma 1. There is a complex-valued continuous function $\Lambda$ in $\mathbb{R}$, periodic of period $2 \pi$, such that $A=\Lambda^{2}$. If $A(\theta) \neq 0$ for every $\theta \in \mathbb{R}$ or if the equation $A(\theta)=0$ has only double roots, then $\Lambda$ is of class $\mathcal{C}^{\omega}$. If $A(\theta)=0$ has single roots, then both $\Lambda^{-1}$ and the distribution derivative of $\Lambda$ belong to $L^{p}(0,2 \pi), 1 \leq p<2$.

Proof. We leave aside the trivial case when $A \equiv 0$. First suppose $\mathfrak{E}(A)$ is contained in a closed half-plane $\xi x+\eta y \geq 0,(\xi, \eta) \in \mathbb{R}^{2}$. There is a branch of the logarithm function defined on $\mathfrak{E}(A)$ and the powers $A(\theta)^{\lambda}=\exp (\lambda \log A(\theta))$ are well-defined, whatever $\lambda \in \mathbb{C}$. If moreover $\mathbb{E}(A) \subset \mathbb{C} \backslash\{0\}$, they are $\mathcal{C}^{\omega}$ functions in $\mathbb{R}$. To say that $\theta^{\circ} \in[0, \pi)$ is a double root of $A(\theta)=0$ is the same as saying that $A(\theta)=\gamma \sin ^{2}\left(\theta-\theta^{\circ}\right)$; it then suffices to fix the determination of $\sqrt{\gamma}$ and to define $\sqrt{A(\theta)}=\sqrt{\gamma} \sin \left(\theta-\theta^{\circ}\right)$. In this case too, $\sqrt{A}$ is an analytic function of $\theta$. Let $\theta^{\circ} \in[0, \pi)$ be a simple root of $A(\theta)=0$. With the determination of $\sqrt{A}$ selected we have $\sqrt{|A(\theta)|}=p(\theta)\left|\theta-\theta^{\circ}\right|^{\frac{1}{2}}$ in a suitably small interval $\left|\theta-\theta^{\circ}\right|<\varepsilon$, with $p>0$ and smooth. The claims about $\Lambda^{-1}$ and $\Lambda^{\prime}$ are obvious consequences of this 
fact. Finally suppose that (2.6) holds. Since here $A(\theta) \neq 0$ for all $\theta \in \mathbb{R}$ there is no problem in defining the function in the real line,

$$
\Lambda(\theta)=\sqrt{|A(\theta)|} \exp \left(\frac{1}{2} i \arg A(\theta)\right) .
$$

Since $A(\theta+\pi)=A(\theta)$ we have

$$
\arg A(\theta+2 \pi)=\arg A(\theta+\pi)+2 k \pi=\arg A(\theta)+4 k \pi
$$

$(k \in \mathbb{Z})$ and therefore

$$
\Lambda(\theta+2 \pi)=\sqrt{|A(\theta)|} \exp \left(\frac{1}{2} i \arg A(\theta)+2 k i \pi\right) .
$$

It follows that $\Lambda(\theta)$ is a $\mathcal{C}^{\omega}$ function in $\mathbb{R}$, periodic of period $2 \pi$.

In what follows we shall write $A^{\frac{1}{2}}$ or $\sqrt{A}$ rather than $\Lambda$; it will always have the meaning as per the proof of Lemma 1 Later we shall need the following.

Lemma 2. If Condition (IN-E) holds, then the winding number of the map $\mathbb{T}^{1} \ni$ $\theta \longrightarrow \sqrt{A(\theta)}$ with respect to the origin is equal to 1 or to -1 , and the range of $\sqrt{A}$ is a curve $\mathfrak{C}_{0}$ without self-intersections.

Proof. Our hypothesis is that the range of $2 A\left(\frac{1}{2} \theta\right), \mathfrak{E}(A)$, winds around $\{0\}$; the square root (2.18), $\sqrt{A}$, is periodic of period $2 \pi$ and therefore $\mathfrak{C}_{0}$ is a curve that also winds around $\{0\}$. If the absolute value of the winding number of $\mathfrak{C}_{0}$ with respect to $\{0\}$ were to exceed 1 , the absolute value of that of $\mathfrak{E}(A)$ would be at least equal to 4 . This would imply that

$$
\frac{1}{2}(b-c)-\frac{1}{2}(b+c) \cos 2 \theta_{j}+\frac{1}{2}(a-d) \sin 2 \theta_{j}=A(0)=-c
$$

for at least three values $0<\theta_{1}<\theta_{2}<\theta_{3}<2 \pi$. In the notation (2.4) this is equivalent to the equations

$$
\alpha \sin \theta_{j} \cos \theta_{j}=\beta \sin ^{2} \theta_{j}, j=1,2,3 .
$$

But (2.6) demands $\operatorname{Im}(\alpha \bar{\beta})>0$, implying $\sin \theta_{j} \cos \theta_{j}=0$; hence $\sin \theta_{j}=0$. Since there is only one zero of $\sin \theta$ in the open interval $(0,2 \pi)$ we have reached a contradiction. We conclude that the winding number of $\mathfrak{C}_{0}$ with respect to $\{0\}$ is equal to \pm 1 . If $\mathfrak{C}_{0}$ had a self-intersection the same would be true of the ellipse $\mathfrak{E}(A)$, an absurdity.

Corollary 2. If Condition (IN-E) holds, then

$$
\lim _{\varepsilon \longrightarrow+0}(\arg A(\theta+\pi-\varepsilon)-\arg A(\theta))= \pm 2 \pi .
$$

Proof. According to Proposition 2, (IN-E) is equivalent to the property that $\partial_{\theta} \arg A(\theta)$ never vanishes. The nonzero number

$$
\frac{1}{2 \pi} \int_{0}^{\pi} \partial_{\theta}(\arg A(\theta)) d \theta=\frac{1}{2 \pi} \int_{0}^{2 \pi} \partial_{\theta}(\arg \sqrt{A(\theta)}) d \theta
$$

is the winding number of the map $\mathbb{T}^{1} \ni \theta \longrightarrow \sqrt{A(\theta)}$ with respect to 0 . 
2.3. The invariant $K(\pi)$. In this subsection we reason under Hypothesis (NOE). This allows us to consider the (analytic) function on the real line,

$$
K(\theta)=\frac{1}{2 \pi i} \int_{0}^{\theta} \frac{d t}{A(t)} .
$$

We have, by (2.3),

$$
\begin{aligned}
K(\pi) & =\frac{1}{2 \pi i} \int_{0}^{2 \pi} \frac{d \theta}{2 A\left(\frac{1}{2} \theta\right)} \\
& =\frac{1}{2 \pi i} \int_{0}^{2 \pi} \frac{d \theta}{\alpha \sin \theta-\beta \cos \theta+\gamma} \\
& =\frac{1}{\pi i} \oint_{|z|=1} \frac{d z}{D(z)}
\end{aligned}
$$

with

$$
D(z)=(\alpha-i \beta) z^{2}+2 i \gamma z-(\alpha+i \beta)
$$

\section{Theorem 6.}

(a): Property $(\boldsymbol{I} \boldsymbol{N}-\boldsymbol{E})$ implies $K(\pi)=0$.

(b): Property (OUT-E) implies $K(\pi) \neq 0$.

When (OUT-E) holds the value $K(\pi)$ is invariant under linear changes of coordinates.

Proof.

Case I (IN-E). If $\alpha=i \beta$ and $\gamma=0$, then $D(z)=-2 \alpha$ and obviously $K(\pi)=0$. If $\alpha=i \beta$ and $\gamma \neq 0$, then $D(z)=2 i \gamma\left(z-\frac{\beta}{\gamma}\right)$ and (2.6) implies $|\beta|>|\gamma|$. We conclude that $\oint_{|z|=1} \frac{d z}{D(z)}=0$. Now suppose $\alpha \neq i \beta$. We observe that (2.6) remains true if we substitute $t \gamma$ for $\gamma$ provided $0 \leq t \leq 1$. We introduce the function

$$
D(z, t)=(\alpha-i \beta) z^{2}+2 i t \gamma z-(\alpha+i \beta), t \in[0,1] .
$$

When $z=e^{i \theta}(\theta \in \mathbb{R})$ we have

$$
D(z, t)=2 i z(\alpha \sin \theta-\beta \cos \theta+t \gamma) .
$$

Proposition 2 and (2.6) ensure that $\alpha \sin \theta-\beta \cos \theta+t \gamma \neq 0$ for all $\theta \in \mathbb{R}$ and $t \in[0,1]$. In other words, no zero of $D(z, t)$ lies on the unit circle whatever $t \in[0,1]$, implying that the number of zeros of $D(z, t)$ in the open unit disk $\Delta$ is independent of $t$. Since

$$
D(z, 0)=-(\alpha-i \beta)\left(z^{2}-\frac{\alpha+i \beta}{\alpha-i \beta}\right)
$$

the absolute values of the roots of $D(z, 0)=0$ are both equal to $\left|\frac{\alpha+i \beta}{\alpha-i \beta}\right|$ and therefore either both roots lie in $\mathbb{C} \backslash \Delta$ or they both lie in $\Delta$ (and are both equal to zero when $\alpha=-i \beta$ ). In all cases (by the sum of residues theorem) we conclude that $\oint_{|z|=1} \frac{d z}{D(z)}=0$. This completes the proof of $(\mathbf{a})$. 
Case II (Case (OUT-E)). As shown in the proof of Theorem 5. (OUT-E) is equivalent to the fact that, after a linear change of the coordinates $x, y,(2.17)$ is valid:

$$
A(\theta)=\frac{1}{2}(\gamma-\beta \cos 2 \theta),
$$

either with $\operatorname{Im}(\beta \bar{\gamma}) \neq 0$, in which case $\mathfrak{E}(A) \subset \mathbb{C} \backslash\{0\}$ is a true ellipse, or with $\beta=\rho \gamma, \rho \in(-1,1)$ [needless to say, $\gamma \neq 0$ in both cases]. If (2.5) holds, after a rotation we can take $\phi=\frac{1}{2} \pi$ and here also we end up with (2.22), which implies

$$
K(\pi)=\frac{1}{2 \pi i} \int_{0}^{2 \pi} \frac{d \theta}{\gamma-\beta \cos \theta}=\frac{1}{\pi} \oint_{|z|=1} \frac{d z}{D^{b}(z)}
$$

with $D^{\mathrm{b}}(z)=\beta z^{2}-2 \gamma z+\beta$. When $\beta=0$ we get

$$
K(\pi)=\frac{1}{i \gamma} .
$$

When $\beta \neq 0$ we exploit the fact that (2.22) and the property $|\beta|<|\gamma|$ remain valid if we substitute $t \beta$ for $\beta$ provided $0 \leq t \leq 1$. We introduce

$$
D^{b}(z, t)=t \beta z^{2}-2 \gamma z+t \beta ;
$$

we know that $D^{b}\left(e^{i \theta}, t\right)=-2 e^{-i \theta}(\gamma-t \beta \cos \theta)$ never vanishes on the unit circle. It follows that the root of $D^{\mathrm{b}}(z, t)=0$,

$$
z_{+}(t)=(t \beta)^{-1} \gamma\left(1+\sqrt{1-t^{2} \beta^{2} \gamma^{-2}}\right)
$$

(with the main branch of the square root), remains in the region $|z|>1$ as $t$ ranges over $(0,1]$, while the root

$$
z_{-}(t)=(t \beta)^{-1} \gamma\left(1-\sqrt{1-t^{2} \beta^{2} \gamma^{-2}}\right) \approx \frac{1}{2} t \beta \gamma^{-2}+O\left(t^{3}\right)
$$

stays inside the open unit disk. Since we have

$$
\frac{1}{D^{b}(z, t)}=-\frac{1}{2} \frac{1}{\sqrt{\gamma^{2}-t^{2} \beta^{2}}}\left(\frac{1}{z-z_{-}(t)}-\frac{1}{z-z_{+}(t)}\right)
$$

we obtain

$$
\frac{1}{\pi} \oint_{|z|=1} \frac{d z}{D(z, t)}=-\frac{1}{2 \pi} \frac{1}{\sqrt{\gamma^{2}-t^{2} \beta^{2}}} \oint_{|z|=1} \frac{d z}{z-z_{-}(t)},
$$

whence, by going to the limit $t=1$,

$$
K(\pi)=\frac{1}{i \sqrt{\gamma^{2}-\beta^{2}}} .
$$

We point out that (2.24) coincides with (2.23) when $\beta=0$. This proves (b).

Case III (Invariance of $K(\pi)$ under linear change of variables $x, y$ ). Each one of the properties (IN-E) and (OUT-E) is invariant, by Corollary 1 . Since we always have $K(\pi)=0$ when (IN-E) is valid it suffices to limit our attention to the class (OUT-E). We reason momentarily in the same coordinate system in which we have proved (2.24). We note that $\gamma^{2}-\beta^{2}=(b-c)^{2}-(b+c)^{2}=-4 b c$, and thus (2.24) can be rewritten as

$$
K(\pi)=-\frac{1}{2 \sqrt{b c}} .
$$


Let $\Gamma \in \mathbf{G L}(2, \mathbb{R})$; we look at the effect of the transformation $\mathfrak{m}(L) \longrightarrow \Gamma^{-1} \mathfrak{m}(L) \Gamma$

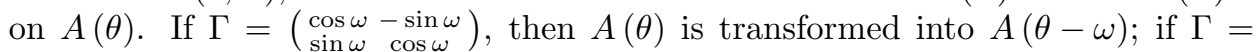
$\left(\begin{array}{cc}\cos \omega & \sin \omega \\ \sin \omega & -\cos \omega\end{array}\right)$, then $A(\theta)$ is transformed into $-A(\omega-\theta)$. None of these transformations affects $K(\pi)$. The dilations $(x, y) \longrightarrow(t x, t y)$ leave $L$ and therefore $A$ unchanged. The stretching $(x, y) \longrightarrow\left(\rho x, \rho^{-1} y\right), 0 \neq \rho \in \mathbb{R}$, transforms $b$ into $b \rho^{-2}$ and $c$ into $c \rho^{2}$, leaving (2.25) unchanged.

2.4. Hypoellipticity, ellipticity and local solvability conditions in $\mathbb{R}^{2} \backslash\{0\}$. In studying the vector field $L$ in $\mathbb{R}^{2} \backslash\{0\}$ we must invoke concepts and results from the general theory of first-order linear differential operators. For the definition of solvability we refer the reader to the very beginning of this article. We recall that $L$ is said to be hypo-elliptic in an open set $\Omega \subset \mathbb{R}^{2}$ if, given any open subset $U$ of $\Omega$ and any distribution $u \in \mathcal{D}^{\prime}(\Omega), L u \in \mathcal{C}^{\infty}(U)$ implies $u \in \mathcal{C}^{\infty}(U)$. The vector field $L$ is said to be elliptic in $\Omega$ if $L$ and $\bar{L}$ are linearly independent at every point of $\Omega$.

We are going to apply to $L$ the characterization of local solvability (see Nirenberg-Treves, 1963]) and and hypo-ellipticity ([Treves, 1971]) for vector fields without critical points. It is convenient to make use of the polar coordinates expression (1.2). We focus on the expression:

$$
\frac{1}{2 i} L \wedge \bar{L}=\frac{1}{2} \operatorname{Im}\left(\left(\operatorname{div} L+A^{\prime}(\theta)\right) \overline{A(\theta)}\right) \partial_{\theta} \wedge r \partial_{r}
$$

To interpret the coefficient of $\partial_{\theta} \wedge r \partial_{r}$ in (2.26) we reason in a sector $\left|\theta-\theta^{\circ}\right|<T$ of $\mathbb{R}^{2} \backslash\{0\}$ in which $A(\theta) \neq 0$. Consider the function of $\theta \in \mathbb{R}$,

$$
\begin{aligned}
W\left(\theta, \theta^{\circ}\right) & =\sqrt{A(\theta)} \exp \left(\frac{1}{2}(\operatorname{div} L) \int_{\theta^{\circ}}^{\theta} \frac{d \tau}{A(\tau)}\right) \\
& =\exp \frac{1}{2}\left((\operatorname{div} L) \int_{\theta^{\circ}}^{\theta} \frac{d \tau}{A(\tau)}+\log A(\theta)\right)
\end{aligned}
$$

with an appropriate choice of the log branch. In this sector,

$$
\arg W\left(\theta, \theta^{\circ}\right)=\frac{1}{2} \operatorname{Im}\left((\operatorname{div} L) \int_{\theta^{\circ}}^{\theta} \frac{d \tau}{A(\tau)}+\log A(\theta)\right)
$$

and we see that $\frac{d}{d \theta} \arg W\left(\theta, \theta^{\circ}\right)$ is equal to the function

$$
\omega^{\prime}(\theta)=\frac{1}{2} \operatorname{Im}\left(\frac{\operatorname{div} L+A^{\prime}(\theta)}{A(\theta)}\right)
$$

whose definition does not depend on $\theta^{\circ}$. We point out that $\omega^{\prime}(\theta)$ is analytic in the region $A(\theta) \neq 0$; it is periodic of period $\pi$ (when looked upon as a possibly unbounded function in $\mathbb{R}$ ). With the notation (2.29) we can rewrite (2.26):

$$
\frac{1}{2 i} L \wedge \bar{L}=|A(\theta)|^{2} \omega^{\prime}(\theta) \partial_{\theta} \wedge r \partial_{r}
$$

By an open sector of $\mathbb{R}^{2} \backslash\{0\}$ we shall mean, in what follows, the radial span of an open arc of the unit circle; the arc and the sector are connected. Our first statement concerns ellipticity and it is an obvious consequence of (2.30):

Proposition 3. The vector field (1.2) is elliptic in an open sector $\Omega$ of $\mathbb{R}^{2} \backslash\{0\}$ if and only if $|A(\theta)| \omega^{\prime}(\theta) \neq 0$ at every point of $\Omega$. 
Corollary 3. If (IN-E) holds and if $\operatorname{div} L=0$, then $L$ is elliptic in the whole of $\mathbb{R}^{2} \backslash\{0\}$.

Proof. This follows directly from Propositions 2 and 3 since $\operatorname{div} L=0 \Longrightarrow$ $\arg W\left(\theta, \theta^{\circ}\right)=\frac{1}{2} \arg A(\theta)$.

It might happen that $L$ is elliptic in the whole of $\mathbb{R}^{2} \backslash\{0\}$ even if (IN-E) does not hold. This is true of the vector field $L=z \partial_{z}=\frac{1}{2}\left(r \partial_{r}-i \partial_{\theta}\right)$ (cf. Example 2 below).

The next statement concerns local solvability; it is borrowed from Treves, 1992, Section VIII.7.

Proposition 4. Suppose that the origin is the sole critical point of the vector field (1.1). Then $L$ is locally solvable in an open sector $\Omega$ of $\mathbb{R}^{2} \backslash\{0\}$ if and only if $\omega^{\prime}(\theta)$ does not change sign at any point of $\Omega$ where $A(\theta) \neq 0$.

If $\operatorname{div} L \neq 0$ and despite (IN-E) holding, $L$ might not even be locally solvable in $\mathbb{R}^{2} \backslash\{0\}$.

Example 1. Take $A(\theta)=\frac{1}{2} \gamma\left(1-2 i e^{-2 i \theta}\right), 0 \neq \gamma \in \mathbb{R}$. Comparing with (2.3) we get $\alpha=-2 \gamma, \beta=-2 i \gamma$; the ellipse $\mathfrak{E}(A)$ is a circle of radius $|\gamma|$ centered at $\frac{1}{2} \gamma$ and therefore (IN-E) is indeed verified. Suppose $a+d=1$. The equation $\omega^{\prime}(\theta)=0$ is equivalent to

$$
\frac{1}{2} \gamma^{-1} \operatorname{Im}\left(A(\theta)\left(1+\overline{A^{\prime}(\theta)}\right)\right)=\gamma \sin 2 \theta-\cos 2 \theta-2 \gamma=0 .
$$

We see that it has two distinct real roots, where $\omega^{\prime}$ changes sign, if $|\gamma|<\frac{1}{\sqrt{3}}$; it has a double root $\left(\right.$ at $\theta=\frac{\pi}{6}$ ) if $\gamma= \pm \frac{1}{\sqrt{3}} ; L$ is elliptic in the whole of $\mathbb{R}^{2} \backslash\{0\}$ for $|\gamma|>\frac{1}{\sqrt{3}}$.

Concerning hypoellipticity the following observation simplifies the picture:

Proposition 5. If the vector field (1.1) is hypoelliptic in $\mathbb{R}^{2} \backslash\{0\}$, then (NO-E) holds.

We recall that (NO-E) is the negation of (ON-E): (NO-E) means that $A(\theta) \neq$ 0 for all $\theta$ and, therefore, that either (IN-E) or (OUT-E) holds. Note also that (NO-E) requires that the origin be the sole critical point of $L$.

Proof. If $A\left(\theta^{\circ}\right)=0$, then $L h=0$, where $h(\theta)=1$ for $\theta^{\circ}<\theta<\theta^{\circ}+\pi, h(\theta)=0$ for all other $\theta$.

We apply the results in ([Treves, 1971):

Proposition 6. Suppose that the vector field (1.2) satisfies Condition (NO-E). Then $L$ is hypoelliptic in an open sector $\Omega$ of $\mathbb{R}^{2} \backslash\{0\}$ if and only if the following two conditions are satisfied:

(he 1) the function $\omega^{\prime}(\theta)$ does not change sign at any point of $\Omega$;

(he 2) the function $\omega^{\prime}(\theta)$ does not vanish identically.

Under Hypothesis (NO-E) we can introduce a primitive $\omega(\theta)$ of $\omega^{\prime}(\theta)$; without further conditions it can only be viewed as a not necessarily periodic function defined in $\mathbb{R}$. The conjunction of Properties (he1)-(he2) can be stated as saying 
that the real $\mathcal{C}^{\omega}$ function $\omega(\theta)$ is strictly monotone in the subset $\left\{\theta \in \mathbb{R} ; e^{i \theta} \in \Omega\right\}$ ("strictly" because the zeros of $\omega^{\prime}$ are isolated). By (2.29) and Definition 1 we have

$$
\omega(\theta+2 k \pi)=\omega(\theta)+2 k \pi \operatorname{Re} \mu(L)
$$

for all $\theta \in \mathbb{R}$ and $k \in \mathbb{Z}$.

Corollary 4. If (NO-E) holds and if $\mu(L) \neq 0$ the following two properties are equivalent:

(1) $L$ is hypoelliptic in $\mathbb{R}^{2} \backslash\{0\}$;

(2) $L$ is locally solvable in $\mathbb{R}^{2} \backslash\{0\}$.

Proof. By (2.31) the hypotheses entail that $\omega^{\prime}$ does not vanish identically, in which case either $\omega^{\prime}$ changes sign, implying (Proposition 4) that $L$ is not locally solvable in $\mathbb{R}^{2} \backslash\{0\}$, or else $\omega^{\prime}$ does not change sign, meaning that $L$ is hypoelliptic (Proposition 6).

Proposition 7. If $L$ is hypoelliptic in $\mathbb{R}^{2} \backslash\{0\}$ and if Hypothesis (OUT-E) is satisfied, then $\operatorname{div} L \neq 0$.

Proof. If $\operatorname{div} L=0$ we have $W\left(\theta, \theta^{\circ}\right)=\sqrt{A(\theta)}$ [cf. (2.27)]. If (OUT-E) holds, the range of $\omega(\theta)=\frac{1}{2} \arg A(\theta)$ is a compact subset of $[0,2 \pi) ; \omega$ cannot be strictly monotone.

Note the contrast between Corollary 3 and Proposition 7.

2.5. Necessity of the conditions in Theorem [3. We begin by relating the Meziani invariant (Definition 1) to the classification in Subsection 2.1. Obviously $\mu(L)=\infty$ defines the class (ON-E). We limit our attention to the cases (NO-E), for which the interpretation of the Meziani invariant in terms of the invariant $K(\pi)$ [see (2.20] ] is clear:

$$
\mu(L)=K(\pi) \operatorname{div} L+\frac{1}{2 i \pi} \lim _{\varepsilon \rightarrow+0}(\log A(\pi-\varepsilon)-\log A(0))
$$

with an appropriate branch of the logarithm function [taking into account the fact that $A(0)=-c$ according to (1.3)]. Theorem [6 implies that if $L$ satisfies (IN-E), then (cf. Corollary 2)

$$
\mu(L)=\frac{1}{2 \pi} \lim _{\varepsilon \rightarrow+0}(\arg A(\pi-\varepsilon)-\arg A(0))= \pm 1 .
$$

When $L$ satisfies (OUT-E), in which case the convex hull of the range of $A$ is contained in $\mathbb{C} \backslash\{0\}$, we have

$$
\mu(L)=K(\pi) \operatorname{div} L
$$

We might have $\mu(L)= \pm 1$ for certain vector fields that satisfy (OUT-E).

Example 2. Take $L=2 z \partial_{z}=r \partial_{r}+i \partial_{\theta}$; thus $A(\theta)=-i$ and $\operatorname{div} L=2$. We have $K(\pi)=\frac{1}{2}$ and $\mu(L)=1$.

The invariance of $\mu(L)$ under linear changes of variables ensues from the invariance of $K(\pi)$ (Theorem [6) and of div $L$. Actually, a stronger result was proved in Meziani, 2001 (Lemma 2.1), where the invariant $\mu(L)$ was first introduced. It applied to a general vector field of class $\mathcal{C}^{\infty}$ in $\mathbb{R}^{2}, L+x^{2} L_{11}+x y L_{12}+y^{2} L_{22}$, where the linear vector field $L$ has the expression (1.1) while the $L_{i j}$ are smooth complex vector fields. The article Meziani, 2001 gives a simple proof of the fact that $\mu(L)$ 
is invariant under diffeomorphisms preserving the origin. The derivation, by perturbation, of the same result from the invariance of $\mu(L)$ under linear changes of variables is also very easy.

Proposition 8. The property $\mu(L)=0$ is equivalent to the conjunction of the properties $(\boldsymbol{O} \boldsymbol{U} \boldsymbol{T}-\boldsymbol{E})$ and $\operatorname{div} L=0$.

Proof. Since $\mu(L)=1$ in the class (IN-E) and $\mu(L)=\infty$ in the class (ON-E) we see that $\mu(L)=0$ entails (OUT-E). By Theorem 6 and (2.33), (OUT-E) and $\mu(L)=0$ demand $\operatorname{div} L=0$, which in turn implies $\mu(L)=0$.

We are now in a position to prove the necessity of the conditions in Theorem 3 ,

Theorem 7. Suppose that the origin is the sole critical point of the vector field (1.1). If $\mu(L)=0$, then $L$ is not locally solvable in any neighborhood of the origin.

Proof. If $\operatorname{div} L=0$ we have

$$
-L=A(\theta) \partial_{\theta}-\frac{1}{2} A^{\prime}(\theta) r \partial_{r} .
$$

First suppose $\arg A(\theta)$ is not a constant; in this case, $z=A(\theta)$ describes an ellipse contained in a half-space $\operatorname{Re}(\bar{z} \zeta)>0(0 \neq \zeta \in \mathbb{C})$. There will be at least one point $\theta^{\circ} \in[0,2 \pi)$ at which $\partial_{\theta} \arg A(\theta)$ changes sign, implying that $L$ is not locally solvable at any point $r e^{i \theta^{\circ}}$ (Proposition 4). Now suppose $A(\theta)=p(\theta) e^{i \theta^{\circ}}$ with $p(\theta) \geq c_{\circ}>0$ for all $\theta$. After multiplication by $e^{-i \theta^{\circ}}$ we may assume that $L$ is a real vector field such that $a+d=0$ and

$$
\forall \theta \in \mathbb{R}, b \sin ^{2} \theta+2 a \cos \theta \sin \theta-c \cos ^{2} \theta>0 .
$$

This means that $a^{2}-b c=\operatorname{det} \mathfrak{m}(L)>0$ and therefore the eigenvalues of $\mathfrak{m}(L)$ are $\pm i \eta, 0 \neq \eta \in \mathbb{R}$. According to Theorem 4 this ensures the nonsolvability of $L$.

The last part of the proof of Theorem 7, combined with Proposition 1, proves the following statement:

Proposition 9. If the vector field (1.1) is locally solvable in $\mathbb{R}^{2} \backslash\{0\}$, then the property $\mu(L)=0$ is equivalent to the negation of Property (ii) in Theorem 1 , Part (B).

\section{Solvability in the Classes (NO-E)}

This section and the next one are entirely devoted to the proof of the sufficiency of the conditions in Part (B) of Theorem 1 and in Theorem 3. As before we assume that the vector field $L$ is given by (1.1) or (1.2); $\mu(L)$ denotes its Meziani invariant (Definition 1). We recall the following two aspects of the classes (NO-E):

Fact 1: Local solvability in $\mathbb{R}^{2} \backslash\{0\}$ is equivalent to hypoellipticity in $\mathbb{R}^{2} \backslash\{0\}$ (Corollary 4).

Fact 2: If $L$ is not elliptic in $\mathbb{R}^{2} \backslash\{0\}$, hypoellipticity in $\mathbb{R}^{2} \backslash\{0\}$ requires $\operatorname{div} L \neq 0$ (Corollary 3 and Proposition 7). 
3.1. Cases (NO-E). Preparatory lemmas. When (IN-E) holds $K(\theta)$ as given in (2.19) is a periodic function of period $\pi$ and therefore can be regarded as an $L^{\infty}$ function (denoted by $K$ ) in $\mathbb{R}^{2}$ homogeneous of degree zero and analytic in $\mathbb{R}^{2} \backslash\{0\}$. Also note that, according to (1.3),

$$
r^{2} A(\theta)=b y^{2}+(a-d) x y-c x^{2} .
$$

If (OUT-E) holds, the range of $A(\theta)$ is contained in a convex cone and, as a consequence, the function $\log A(\theta)$ is well-defined (by selecting an appropriate branch of the logarithm function) as a periodic function of period $2 \pi$ and class $\mathcal{C}^{\omega}$.

Lemma 3. If (IN-E) holds, then $L(2 i \pi K)=-1$. If (OUT-E) holds and if $\operatorname{div} L \neq 0$, then

$$
L\left(\frac{\log \left(r^{2} A(\theta)\right)}{\operatorname{div} L}\right)=1 .
$$

The equations in Lemma 3 are to be understood in the distribution sense.

Proof. We rely on the expression (1.2) of $L$. When (IN-E) holds it is evident that $L K=-A \partial_{\theta} K=-(2 i \pi)^{-1}$. Now suppose (OUT-E) holds. We have

$$
\begin{gathered}
\frac{1}{2}\left(\operatorname{div} L+A^{\prime}\right) r \partial_{r}\left((\operatorname{div} L)^{-1}(2 \log r+\log A)\right)=1+(\operatorname{div} L)^{-1} A^{\prime}, \\
A \partial_{\theta}\left(\tau^{-1} \log \left(r^{2} A\right)\right)=(\operatorname{div} L)^{-1} A^{\prime},
\end{gathered}
$$

implying (3.18) directly.

Remark 5. We see that, under the hypotheses of Lemma $3 L$ is not hypoelliptic in $\mathbb{R}^{2}$ since neither $K$ nor $\log \left(r^{2} A(\theta)\right)$ is smooth at the origin. Propositions 5 and 7 state that, if (ON-E) holds, or if (OUT-E) holds and $\operatorname{div} L=0$, then $L$ is not hypoelliptic in $\mathbb{R}^{2} \backslash\{0\}$. We conclude that no vector field (1.1) is hypoelliptic in $\mathbb{R}^{2}$.

Lemma 4. Let $\zeta \in \mathbb{C}$ be such that $\operatorname{Re} \zeta>0$ and let $m$ be a positive integer. Suppose that $\Psi(r, \theta) \in \mathcal{C}^{\infty}\left(\mathbb{R}_{+} \times \mathbb{R}\right)$ with $\mathbb{R}_{+}=[0,+\infty)$ is periodic (in the angular variable $\theta)$ of period $2 \pi$ and vanishes for large $r$. If there is a constant $C>0$ such that

$$
\forall(r, \theta) \in \mathbb{R}_{+} \times \mathbb{R},|\Psi(r, \theta)|+\left|\partial_{\theta} \Psi(r, \theta)\right| \leq C r^{m},
$$

then there is a (distribution) solution $\Phi(r, \theta) \in \mathcal{C}\left(\mathbb{R}_{+} \times \mathbb{R}\right)$ of the equation

$$
\zeta \partial_{\theta} \Phi-i r \partial_{r} \Phi=\Psi
$$

in $\mathbb{R}_{+} \times \mathbb{R}$, periodic of period $2 \pi$ with respect to $\theta$ and such that $|\Phi(r, \theta)| \leq$ $C^{\prime} r^{m}(1+|\ln r|)$ for some constant $C^{\prime}>0$ and all $(r, \theta) \in \mathbb{R}_{+} \times \mathbb{R}$.

Proof. Suppose $z=r e^{i \theta} \in \operatorname{supp} \Psi \Longrightarrow r<R$. We use Fourier series expansions:

$$
\Psi=\sum_{k=-\infty}^{+\infty} \psi_{k}(r) e^{i k \theta}, \Phi=\sum_{k=-\infty}^{+\infty} \varphi_{k}(r) e^{i k \theta} .
$$

The standard formula for the Fourier coefficients shows that $r \geq R \Longrightarrow \psi_{k}(r)=0$; (3.3) implies

$$
(1+|k|)\left|\psi_{k}(r)\right| \leq C_{1} r^{m}
$$

with $C_{1}>0$ independent of $k \in \mathbb{Z}$ and of $r \geq 0$; (3.4) requires

$$
\left(r \partial_{r}+i \zeta \partial_{\theta}\right)\left(\Phi e^{-i k \theta}\right)-k \zeta \Phi e^{-i k \theta}=i \Psi e^{-i k \theta},
$$


whence, by integration with respect to $\theta$ over $[0,2 \pi]$,

$$
r \partial_{r} \varphi_{k}-k \zeta \varphi_{k}=r^{1+k \zeta} \partial_{r}\left(r^{-k \zeta} \varphi_{k}\right)=i \psi_{k} .
$$

We select

$$
\begin{aligned}
& \varphi_{k}(r)=i \int_{0}^{r}(t / r)^{-k \zeta} \psi_{k}(t) \frac{d t}{t} \text { if } k \operatorname{Re} \zeta<m, \\
& \varphi_{k}(r)=-i \int_{r}^{R}(r / t)^{k \zeta} \psi_{k}(t) \frac{d t}{t} \text { if } k \operatorname{Re} \zeta \geq m .
\end{aligned}
$$

We apply (3.5) to get, for $k \operatorname{Re} \zeta<m$,

$$
\begin{aligned}
\left|\varphi_{k}(r)\right| & \leq C_{1}(1+|k|)^{-1} r^{m-1} \int_{0}^{r}(t / r)^{m-1-k \operatorname{Re} \zeta} d t \\
& =C_{1} r^{m}(1+|k|)^{-1}(m-k \operatorname{Re} \zeta)^{-1}
\end{aligned}
$$

and for $k \operatorname{Re} \zeta>m$,

$$
\begin{aligned}
\left|\varphi_{k}(r)\right| & \leq C_{1}(1+|k|)^{-1} r^{m-1} \int_{r}^{R}(r / t)^{k \operatorname{Re} \zeta-m+1} d t \\
& =C_{1}(1+|k|)^{-1} r^{m} \int_{1}^{R / r} t^{-k \operatorname{Re} \zeta+m-1} d t \\
& \leq C_{1}(1+|k|)^{-1}(k \operatorname{Re} \zeta-m)^{-1} r^{m} .
\end{aligned}
$$

If $k \operatorname{Re} \zeta=m$ we have

$$
\begin{aligned}
\left|\varphi_{k}(r)\right| & \leq C_{1}\left(1+m|\operatorname{Re} \zeta|^{-1}\right)^{-1} r^{m} \int_{1}^{R / r} t^{-1} d t \\
& =C_{1}\left(1+m|\operatorname{Re} \zeta|^{-1}\right)^{-1} r^{m}(\ln R-\ln r) .
\end{aligned}
$$

From all this we derive, for a suitably large constant $C_{2}>0$ and all $k \in \mathbb{Z}$,

$$
\sup _{r>0}\left|\varphi_{k}(r)\right| \leq C_{2}(1+|k|)^{-2} r^{m}(1+|\ln r|),
$$

directly implying the claim.

For use in the next two subsections we state

Corollary 5. Let $m$ be a positive integer. If $\Psi(r, \theta) \in \mathcal{C}_{c}^{1}\left(\mathbb{R}_{+} \times \mathbb{R}\right)$ is periodic in the angular variable $\theta$ of period $2 \pi T(T>0)$ and satisfies (3.3) for some constant $C>0$, then there is a distribution solution $\Phi(r, \theta) \in \mathcal{C}\left(\mathbb{R}_{+} \times \mathbb{R}\right)$ of the equation

$$
\partial_{\theta} \Phi-i r \partial_{r} \Phi=\Psi
$$

in $\mathbb{R}_{+} \times \mathbb{R}$, periodic of period $2 \pi T$ and such that $|\Phi(r, \theta)| \leq C^{\prime} r^{m}(1+|\ln r|)$ for some constant $C^{\prime}>0$ and all $(r, \theta) \in \mathbb{R}_{+} \times \mathbb{R}$.

Proof. The change of angular variable $\theta \longrightarrow T^{-1} \theta$ transforms (3.6) into (3.4) in which $\zeta=T^{-1}$ and $\Psi$ is replaced by $T^{-1} \Psi$. 
3.2. Vector field elliptic in $\mathbb{R}^{2} \backslash\{0\}$. Case $\mu(L) \in \mathbb{R}$. We return to (1.2)-(1.3); under Hypothesis (NO-E) we may consider the differential operator in $\mathbb{R}^{2} \backslash\{0\}$,

$$
-A(\theta)^{-1} L=\partial_{\theta}-\frac{a+d+A^{\prime}(\theta)}{2 A(\theta)} r \partial_{r} .
$$

We are going to make use of the function (2.27) with the choice $\theta^{\circ}=0$; we define

$$
\begin{aligned}
W(\theta) & =\sqrt{A(\theta)} \exp \left(\frac{1}{2}(\operatorname{div} L) \int_{0}^{\theta} \frac{d \tau}{A(\tau)}\right) \\
& =\exp \frac{1}{2}\left((\operatorname{div} L) \int_{0}^{\theta} \frac{d \tau}{A(\tau)}+\log A(\theta)\right) .
\end{aligned}
$$

According to Definition 1 we have

$$
W(2 \pi)=\exp (2 i \pi \mu(L)) .
$$

If we assume that $\mu(L) \in \mathbb{R}$ we can get an expression for $L$ simpler than (3.7). Indeed, it allows us to introduce the new "radial" variable

$$
\rho=r|W(\theta)|=r \exp \frac{1}{2} \operatorname{Re}\left((\operatorname{div} L) \int_{0}^{\theta} \frac{d \tau}{A(\tau)}+\log A(\theta)\right) .
$$

According to (3.9), $\rho$ is a periodic function of period $2 \pi$ of $\theta$; the change of variables $(r, \theta) \rightarrow(\rho, \theta)$ "rectifies" the vector field $-\operatorname{Re}\left(A(\theta)^{-1} L\right)$. In the coordinates $(\rho, \theta)$ it becomes $\partial_{\theta}$ (note that $r \partial_{r}=\rho \partial_{\rho}$ ) and (3.7) simplifies to

$$
-A(\theta)^{-1} L=\partial_{\theta}-i \omega^{\prime}(\theta) \rho \partial_{\rho}
$$

Theorem 8. Suppose that the vector field (1.1) is elliptic and that $\mu(L) \in \mathbb{R} \backslash\{0\}$. Under these hypotheses, to every $F \in \mathcal{C}_{c}^{1}\left(\mathbb{R}^{2}\right)$ there is a distribution solution $u$ of the equation $L u=F$ in $\mathbb{R}^{2}$ such that $u+2 i \pi F(0) K$ in the (IN-E) case, $u-$ $F(0)(\operatorname{div} L)^{-1} \log \left(r^{2} A\right)$ in the (OUT-E) case, is a continuous function in $\mathbb{R}^{2}$ that vanishes at the origin.

Recall that $\mu(L) \neq 0 \Longleftrightarrow \operatorname{div} L \neq 0$ in the (OUT-E) case.

Proof. The hypothesis $\mu(L) \in \mathbb{R}$ allows us to make use of the expression (3.11). We see that, given any $F \in \mathcal{C}_{\mathrm{c}}^{1}\left(\mathbb{R}^{2}\right)$, the equation $L u=F$ is equivalent to

$$
\partial_{\theta} u-i \omega^{\prime}(\theta) \rho \partial_{\rho} u=-A^{-1}(\theta) F(\rho, \theta) \text {. }
$$

We shall assume that $F(\rho, \theta)=0$ if $\rho>R(0<R<+\infty)$. To solve (3.12) there is no loss of generality in assuming that $F(0)=0$. Indeed, it suffices to solve

$$
L v=F-F(0) \chi(\rho)-F(0) u_{\circ} L \chi(\rho),
$$

where:

(1) $u_{\circ}=-2 i \pi K$ if (IN-E) holds and $u_{\circ}=(\operatorname{div} L)^{-1} \log \left(r^{2} A\right)$ if (OUT-E) holds (Lemma 3);

(2) $\chi \in \mathcal{C}^{\infty}(\mathbb{R}), \chi(\rho)=1$ for $\rho<\frac{1}{3} R$ and $\chi(\rho)=0$ for $\rho>\frac{2}{3} R$.

The right-hand side in (3.13) is a $\mathcal{C}^{1}$ function in $\mathbb{R}^{2}$ that vanishes at the origin $(L \chi$ vanishes identically in the disk $\rho<\frac{1}{3} R$ ). We can then take

$$
u=v+F(0) \chi(\rho) u_{\circ} .
$$


The ellipticity of $L$ in $\mathbb{R}^{2} \backslash\{0\}$ means that $\omega^{\prime}(\theta) \neq 0$ for every $\theta \in \mathbb{R}$ (Proposition 3). The map $\mathbb{R} \ni \theta \longrightarrow \omega(\theta) \in \mathbb{R}$ is analytic and strictly monotone; let us assume that it is increasing. We apply Formula (2.31) and its consequence, $\theta(\omega+2 \pi T)=$ $\theta(\omega)+2 \pi$, where $T=\mu(L)$. The change of variables $(\rho, \theta) \longrightarrow(\rho, \omega)$ maps bijectively the cylinder $\Gamma_{1}=\mathbb{R}_{+} \times(\mathbb{R} / 2 \pi \mathbb{Z})$ onto the cylinder $\Gamma_{T}=\mathbb{R}_{+} \times(\mathbb{R} / 2 \pi T \mathbb{Z})$; it is a diffeomorphism between real-analytic manifolds with boundary. If $\varphi \in \mathcal{C}_{\mathrm{c}}^{\infty}\left(\mathbb{R}^{2}\right)$ we continue to denote by $\varphi$ its pullback under the natural projection $\Gamma_{1} \ni(r, \theta) \longrightarrow$ $(r \cos \theta, r \sin \theta) \in \mathbb{R}^{2}$. We can view $-A(\theta)^{-1} L$ as a differential operator $L_{1}$ on $\Gamma_{1}$ and consider, for some $u \in \mathcal{C}\left(\Gamma_{1}\right)$,

$$
\begin{aligned}
\left\langle L_{1} u, \varphi\right\rangle & =\iint_{\Gamma_{1}} u\left(L_{1}^{\top} \varphi\right) \rho d \rho d \theta \\
& =-\iint_{\Gamma_{1}} u\left(\partial_{\theta} \varphi-i \omega^{\prime}(\theta) \rho \partial_{\rho} \varphi\right) \rho d \rho d \theta+2 i \iint_{\Gamma_{1}} u \varphi \omega^{\prime}(\theta) \rho d \rho d \theta .
\end{aligned}
$$

Since $d \omega=\omega^{\prime}(\theta) d \theta, \partial_{\theta}=\omega^{\prime}(\theta) \partial_{\omega}$, we get

$$
\left\langle L_{1} u, \varphi\right\rangle=-\iint_{\Gamma_{T}} u_{*}\left(\partial_{\omega} \varphi_{*}-i \rho \partial_{\rho} \varphi_{*}-2 i \varphi_{*}\right) \rho d \rho d \omega,
$$

where $u_{*}(\rho, \omega)=u(\rho, \theta(\omega)), \varphi_{*}(\rho, \omega)=\varphi(\rho, \theta(\omega))$ [here $\theta(\omega) \in \mathbb{R} / 2 \pi \mathbb{Z}$ ]. Then let $G_{*}(\rho, \omega)$ denote the pushforward to $\Gamma_{T}$ of the function $-\omega^{\prime}(\theta)^{-1} A(\theta)^{-1} F(\rho, \theta)$ under the diffeomorphism $(\rho, \theta) \longrightarrow(\rho, \omega)$. Thanks to Corollary 5 we can select $u_{*} \in \mathcal{C}\left(\Gamma_{T}\right)$ to be a distribution solution of the equation $\partial_{\omega} u_{*}-i \rho \partial_{\rho} u_{*}=G_{*}$ in $\Gamma_{T}$ such that $\left|u_{*}(\rho, \omega)\right| \leq$ const. $\rho(1+|\ln \rho|)$ for all $(\rho, \omega) \in \Gamma_{T}$. This means that

$$
\iint_{\Gamma_{T}} G_{*} \varphi_{*} \rho d \rho d \omega=-\iint_{\Gamma_{T}} u_{*}\left(\partial_{\omega} \varphi_{*}-i \rho \partial_{\rho} \varphi_{*}-2 i \varphi_{*}\right) \rho d \rho d \omega
$$

We have

$$
\iint_{\Gamma_{T}} G_{*} \varphi_{*} \rho d \rho d \omega=-\iint_{\Gamma_{1}} A(\theta)^{-1} F \varphi \rho d \rho d \theta .
$$

Combining (3.15), (3.16) and (3.17) yields $L_{1} u=-A(\theta)^{-1} F$. The distribution solution $u$ is a continuous function in $\mathbb{R}^{2}$ such that $|u(r, \theta)| \leq$ const. $r(1+|\ln r|)$ for all $(r, \theta) \in \mathbb{R}^{2}$.

Corollary 6. The conclusion in Theorem 8 is valid if $L$ satisfies (IN-E) and if $\operatorname{div} L=0$.

Proof. This follows from Corollary 3 .

3.3. Vector field elliptic in $\mathbb{R}^{2} \backslash\{0\}$. Case $\mu(L) \notin \mathbb{R}$. To handle the case of a vector field (1.1) elliptic in $\mathbb{R}^{2} \backslash\{0\}$ and such that $\mu(L) \notin \mathbb{R}$ we avail ourselves of Theorem 2.1, Meziani, 2001, or its generalization, Theorem 1.3 (Cordaro-Gong, 2004). In our situation the latter yields

Lemma 5. Suppose that the vector field (1.1) is elliptic in $\mathbb{R}^{2} \backslash\{0\}$ and that $\mu(L) \notin$ $\mathbb{Q}$. Then, for each positive integer $k$ there is a $C^{k}$ diffeomorphism $U \longrightarrow V$, with $U$ and $V$ neighborhoods of $\{0\} \times \mathbb{S}^{1} \subset \mathbb{R} \times \mathbb{S}^{1}$, preserving $\{0\} \times \mathbb{S}^{1}$ and transforming $L$ into $\kappa(r, \theta)\left(\partial_{\theta}-i \mu(L) r \partial_{r}\right)$, with both $\kappa(r, \theta)$ and $\kappa(r, \theta)^{-1}$ belonging to $L^{\infty}$ and smooth for $r>0$. 
We can state

Theorem 9. Suppose that the vector field (1.1) is elliptic in $\mathbb{R}^{2} \backslash\{0\}$ and that $\mu(L) \notin \mathbb{Q}$. If $\Omega$ is a bounded open subset of $\mathbb{R}^{2}$, then to every $F \in \mathcal{C}_{c}^{1}(\Omega)$ there is $u \in \mathcal{D}^{\prime}\left(\mathbb{R}^{2}\right)$ such that $L u=F$ in $\mathbb{R}^{2}$ and such that, moreover, $u-i \mu(L)^{-1} F(0) \log r$ is a continuous function in $\mathbb{R}^{2}$ vanishing at the origin.

Proof. As pointed out in the introduction it suffices to prove the claim for $\Omega$ a neighborhood, however small, of the origin. In view of Lemma 5 it suffices to solve the equation

$$
\partial_{\theta} u-i \mu(L) r \partial_{r} u=F
$$

in the open disk $\Delta_{\varepsilon}=\left\{(x, y) \in \mathbb{R}^{2}: x^{2}+y^{2}<\varepsilon^{2}\right\}(\varepsilon>0$ suitably small) for an arbitrary $F \in \mathcal{C}_{\mathrm{c}}^{\infty}\left(\Delta_{\varepsilon}\right)$. In solving (3.18) there is no loss of generality in assuming that $F(0)=0$ since (3.18) is equivalent to

$$
\left(\partial_{\theta}-i \mu(L) r \partial_{r}\right)\left(u-i \mu(L)^{-1} F(0) \log r\right)=F-F(0) .
$$

Since $L$ is elliptic, and therefore the same is true of $\partial_{\theta}-i \mu(L) r \partial_{r}$, necessarily $\operatorname{Re} \mu(L) \neq 0$; possibly after a reorientation $\theta \longrightarrow-\theta$ we may assume $\operatorname{Re} \mu(L)>0$. Theorem 9 is then a direct consequence of Lemma 4 where we put $\zeta=\mu(L)^{-1}$.

3.4. Vector field hypoelliptic but not elliptic in $\mathbb{R}^{2} \backslash\{0\}$. Case $\mu(L) \in \mathbb{R}$. We begin by extracting from the hypoellipticity hypothesis the information that will be needed. Keep in mind that $A$ is periodic of period $\pi$ and never vanishes. In this subsection $L$ shall not be elliptic in $\mathbb{R}^{2} \backslash\{0\}$ and therefore $\operatorname{div} L=a+d \neq 0$ (Fact 2 at the start of this section).

We refer the reader to the last part of Subsection 3.1 and to Proposition 5 the function

$$
|A(\theta)|^{2} \omega^{\prime}(\theta)=\frac{1}{2} \operatorname{Im}\left(\left(A^{\prime}(\theta)+\operatorname{div} L\right) \overline{A(\theta)}\right)
$$

must have a unique double zero in $[0, \pi)$. This is the same as saying that the quadratic form

$$
\operatorname{Im}((a x+b y)(\bar{c} x+\bar{d} y))=x^{2} \operatorname{Im}(a \bar{c})+x y \operatorname{Im}(a \bar{d}+b \bar{c})+y^{2} \operatorname{Im}(b \bar{d})
$$

is semidefinite and vanishes on a single straight line through the origin (and nowhere else). After a rotation we may assume that this line is the real axis, which is equivalent to the properties

$$
\operatorname{Im}(a \bar{c})=0, \operatorname{Im}(a \bar{d}+b \bar{c})=0, \operatorname{Im}(b \bar{d}) \neq 0 .
$$

Notice that (3.20) demands that $b d \neq 0$; it is convenient to divide $L$ by $d$ and to assume $d=1$, in which case we must have $\operatorname{Im} b \neq 0$. We deduce from (1.3):

$$
\frac{1}{2} \operatorname{Im}\left(\left(a+1+A^{\prime}(\theta)\right) \overline{A(\theta)}\right)=(\operatorname{Im} b) \sin ^{2} \theta
$$

We can state

Lemma 6. Suppose that $L$ is hypoelliptic but not elliptic in $\mathbb{R}^{2} \backslash\{0\}$. After a linear change of the coordinates in $\mathbb{R}^{2}$ we have $\operatorname{Im} b \neq 0$ and $|A(\theta)|^{2} \omega^{\prime}=(\operatorname{Im} b) \sin ^{2} \theta$.

Lemma 7. If $L$ is hypoelliptic but not elliptic in $\mathbb{R}^{2} \backslash\{0\}$, then $\operatorname{det} \mathfrak{m}(L) \neq 0$. 
Proof. If $a=0$, then $\operatorname{det} \mathfrak{m}(L)=b c \neq 0$, a consequence of (NO-E). Assuming that (3.20) holds we see that $a \neq 0 \Longrightarrow c=s a, s \in \mathbb{R}$, and $\operatorname{det} \mathfrak{m}(L)=a(d-s b) \neq 0$ since $b / d \notin \mathbb{R}$.

Lemma 8. Suppose that $L$ is hypoelliptic but not elliptic in $\mathbb{R}^{2} \backslash\{0\}$. To every pair of functions $f_{j}(x) \in \mathcal{C}^{\infty}(\mathbb{R}), j=0,1$, there is $u_{0} \in L_{\text {loc }}^{1}\left(\mathbb{R}^{2}\right)$ such that $L u_{0}-f_{0}(x)-y f_{1}(x)=y^{2} g(x, y)$ with $g \in \mathcal{C}^{\infty}\left(\mathbb{R}^{2}\right)$. We can take

$$
u_{0}(x, y)=-2 \pi i f_{0}(0) K(\theta)+\phi(x) y+\psi(x) y^{2} \text { if (IN-E) holds, }
$$$$
u_{0}(x, y)=-f_{0}(0)(\operatorname{div} L)^{-1} \log \widetilde{A}(x, y)+\phi(x) y+\psi(x) y^{2} \text { if (OUT-E) holds, }
$$

where $\phi, \psi \in \mathcal{C}^{\infty}(\mathbb{R})$.

Proof. Thanks to Lemma 4 it suffices to solve

$$
L v_{0} \cong f_{0}(x)-f_{0}(0)-y f_{1}(x) \bmod y^{2}
$$

and then take

$$
u_{0}=v_{0}-2 \pi i f_{0}(0) K(\theta)
$$

if (IN-E) holds and take

$$
u_{0}=v_{0}-f_{0}(0)(\operatorname{div} L)^{-1} \log \left(r^{2} A\right)
$$

if (OUT-E) holds (we know that $\operatorname{div} L \neq 0$ ). Since

$$
K(\theta) \in L^{\infty}\left(\mathbb{R}^{2}\right) \quad \text { and } \quad \log (r A(\theta)) \in L_{\mathrm{loc}}^{1}\left(\mathbb{R}^{2}\right)
$$

there is no loss of generality in assuming that $f_{0}(0)=0$. We note that

$$
\begin{aligned}
L\left(c^{-1} y x^{-1} f_{0}(x)\right)= & f_{0}(x)+c^{-1} y\left(d x^{-1} f_{0}(x)+a x \partial_{x}\left(x^{-1} f_{0}(x)\right)\right) \\
& +b c^{-1} y^{2} x \partial_{x}\left(x^{-1} f_{0}(x)\right) .
\end{aligned}
$$

The function $\widetilde{f}_{1}(x)=c^{-1}\left(x^{-1} f_{0}(x)+a x \partial_{x}\left(x^{-1} f_{0}(x)\right)\right)+f_{1}(x)$ belongs to $\mathcal{C}^{\infty}(\mathbb{R})$. Lemma 7 enables us to find two complex numbers $\xi, \eta$ such that

$$
(a x+b y) \xi+(c x+d y) \eta=y \widetilde{f}_{1}(0) .
$$

It suffices to solve $L v_{1}=y\left(\widetilde{f}_{1}(x)-\widetilde{f}_{1}(0)\right)+O\left(y^{2}\right)$ and then select $v_{0}=v_{1}+\xi x+$ $\eta y$. We simply take

$$
v_{1}=\frac{1}{2} c^{-1} y^{2} x^{-1}\left(\widetilde{f}_{1}(x)-\widetilde{f}_{1}(0)\right) .
$$

Indeed,

$$
\begin{aligned}
L v_{1}= & y\left(\widetilde{f}_{1}(x)-\widetilde{f}_{1}(0)\right)+d c^{-1} y^{2} x^{-1}\left(\widetilde{f}_{1}(x)-\widetilde{f}_{1}(0)\right) \\
& +\frac{1}{2} c^{-1}(a x+b y) y^{2} \partial_{x}\left(x^{-1}\left(\widetilde{f}_{1}(x)-\widetilde{f}_{1}(0)\right)\right),
\end{aligned}
$$

whence the desired result.

Theorem 10. Suppose that the vector field (1.1) is hypoelliptic but not elliptic in $\mathbb{R}^{2} \backslash\{0\}$ and that $\mu(L) \in \mathbb{R} \backslash\{0\}$.

Under these hypotheses, to every $F \in \mathcal{C}_{c}^{1}\left(\mathbb{R}^{2}\right)$ there is a distribution $u \in \mathcal{D}^{\prime}\left(\mathbb{R}^{2}\right)$ such that $L u=F$ in $\mathbb{R}^{2}$ and such that $u+2 i \pi F(0) K$ in the (IN-E) case, $u-$ $(\operatorname{div} L)^{-1} F(0) \log \left(r^{2} A(\theta)\right)$ in the (OUT-E) case, are continuous functions in $\mathbb{R}^{2}$ vanishing at the origin. 
Proof. In accordance with Lemma $\left[6\right.$ we assume that $|A(\theta)|^{2} \omega^{\prime}=(\operatorname{Im} b) \sin ^{2} \theta$, $\operatorname{Im} b \neq 0$. The hypothesis that $\mu(L) \in \mathbb{R}$ allows us to make use of the expression (3.11) of $-A(\theta)^{-1} L$ : given any $F \in \mathcal{C}_{\mathrm{c}}^{1}\left(\mathbb{R}^{2}\right)$, the equation $L u=F$ is equivalent to (3.11). Lemma 8 shows that there is no loss of generality in assuming $F(\rho, \theta)$ to be divisible by $\rho^{2} \sin ^{2} \theta$ in $\mathcal{C}^{\infty}\left(\mathbb{R}^{2}\right)$; this is equivalent to saying that $\omega^{\prime}(\theta)^{-1} F(\rho, \theta)$ is a (compactly supported) $\mathcal{C}^{1}$ function in $\mathbb{R}^{2}$ vanishing to second order at $\rho=0$. Here also the map $\mathbb{R} \ni \theta \longrightarrow \omega(\theta) \in \mathbb{R}$ is analytic and strictly monotone (but it is not an analytic diffeomorphism). Possibly after a change of orientation $\theta \rightarrow-\theta$ we can assume that $\omega$ is strictly increasing. From here on we repeat almost verbatim the proof of Theorem 9 the essential modification is that the map $(\rho, \theta) \longrightarrow(\rho, \omega)$ is a homeomorphism of $\Gamma_{1}=\mathbb{R}_{+} \times(\mathbb{R} / 2 \pi \mathbb{Z})$ onto $\Gamma_{T}=\mathbb{R}_{+} \times(\mathbb{R} / 2 \pi T \mathbb{Z})$ with a Hölder continuous inverse, and not a diffeomorphism between analytic manifolds with boundary. The function $G_{*}(\rho, \omega)$ is the pushforward to $\Gamma_{T}$ of the function $-\omega^{\prime}(\theta)^{-1} A(\theta)^{-1} F(\rho, \theta)$ under said homeomorphism. Here we also apply Corollary 5 to select a distribution solution $u_{*} \in \mathcal{C}\left(\Gamma_{T}\right)$ of the equation $\partial_{\omega} u_{*}-i \rho \partial_{\rho} u_{*}=G_{*}$ in $\Gamma_{T}$. In the nonelliptic case we get that $\left|u_{*}(\rho, \omega)\right| \leq$ const. $\rho^{2}(1+|\ln \rho|)$ for all $(\rho, \omega) \in \Gamma_{T}$. The equations (6.3), (6.4) and (6.5) remain valid and allow us to conclude that $L_{1} u=-A(\theta)^{-1} F$. The regularity assertions follow directly from Lemma 8 .

Corollary 7. If the vector field (1.1) satisfies (IN-E) and is locally solvable in $\mathbb{R}^{2} \backslash\{0\}$, then it is globally solvable in $\mathbb{R}^{2}$.

Example 3. Putting $a=0, b=c=1, d=\operatorname{div} L=-i$ in (1.1) yields $L=$ $y \partial_{x}+(x+i y) \partial_{y}$, a vector field hypoelliptic in $\mathbb{R}^{2} \backslash\{0\}$ since

$$
\operatorname{Im}((a x+b y)(\bar{c} x+\bar{d} y))=-y^{2} .
$$

The ellipse $\mathfrak{E}(A)$ is defined by the equation $x^{2}+4 y^{2}=1$ and thus $L$ satisfies (IN-E): $\mu(L)=1$. Theorem 10 applies.

Example 4. Putting $a=0, b=-c=1, d=\operatorname{div} L=-i$ in (1.1) yields $L=$ $y \partial_{x}-(x+i y) \partial_{y}$, a vector field hypoelliptic in $\mathbb{R}^{2} \backslash\{0\}$ since

$$
\operatorname{Im}((a x+b y)(\bar{c} x+\bar{d} y))=y^{2} .
$$

The ellipse $\mathfrak{E}(A)$ is the segment $z=1+\frac{1}{2} i \sin \theta$ and thus $L$ satisfies (OUT-E). Using Formula (2.25) one finds $\mu(L)=\frac{1}{2}$. Theorem 10 applies.

3.5. Vector field hypoelliptic but not elliptic in $\mathbb{R}^{2} \backslash\{0\}$. Case $\mu(L) \notin \mathbb{R}$. This subsection is devoted to the proof of the following result:

Theorem 11. Suppose that the vector field (1.1) is hypoelliptic but not elliptic in $\mathbb{R}^{2} \backslash\{0\}$ and that $\mu(L) \in \mathbb{C} \backslash \mathbb{R}$. Under this hypothesis $L$ is locally solvable in $\mathbb{R}^{2}$.

Proof of Theorem 11. Recall that (IN-E) $\Longrightarrow \mu(L)= \pm 1$. Thus, if $L$ is not elliptic but is hypoelliptic in $\mathbb{R}^{2} \backslash\{0\}$ and if $\mu(L) \in \mathbb{C} \backslash \mathbb{R}$ (thus $\mu \neq \infty$ ), then $L$ must satisfy (OUT-E) and $a+d \neq 0$. We return to (3.20)-(3.21). Since (3.20) requires $b d \neq 0$, division by $d$ allows us to assume $d=1$. We must distinguish the two cases $a=0$ and $a \neq 0$. 
3.5.1. Proof of Theorem 11, Case $a=0$. When $a=0$, (3.20) implies $c=t b$ with $t \in \mathbb{R}$, and (3.21) implies $t \operatorname{Im} b \neq 0$ :

$$
L=b y \partial_{x}+(t b x+y) \partial_{y}
$$

We make the change of variables $x \rightarrow|t|^{-\frac{1}{4}} x, y \rightarrow|t|^{\frac{1}{4}} y$ and the change of notation $b \rightarrow b|t|^{-\frac{1}{2}}$, transforming $L$ into

$$
L=b\left(y \partial_{x}+\varepsilon x \partial_{y}\right)+y \partial_{y}, \varepsilon=\frac{t}{|t|} .
$$

We find ourselves in the situation where $a=0, c=\varepsilon b, d=1$ and $\operatorname{Im} b \neq 0$. With these choices (2.9) reads $0<\varepsilon(\operatorname{Im} b)^{2}$, showing that $\varepsilon=+1$ implies (IN-E), a case that is settled (Corollary 7). When $\varepsilon=-1$, Property (OUT-E) holds and

$$
L=y \partial_{y}-b \partial_{\theta} .
$$

According to Formulas (2.25) and (2.33), $\mu(L)=\frac{1}{2 b} i \notin \mathbb{R}$ unless $\operatorname{Re} b=0$.

Proposition 10. The vector field (3.22) is globally solvable in $\mathbb{R}^{2}$.

Proof. Since $L$ is hypoelliptic but not elliptic in $\mathbb{R}^{2} \backslash\{0\}$ we can apply Lemma 8. it suffices to solve the equation $L u=y^{2} G(x, y)$ with $G \in \mathcal{C}_{\mathrm{c}}^{\infty}\left(\mathbb{R}^{2}\right)$ arbitrary. We can handle separately the equations $L u_{+}=H(y) y^{2} G(x, y)$ and $L u_{-}=$ $H(y) y^{2} G(x,-y), H$ being the Heaviside function. Considering (3.22), the existence of continuous solutions $u_{ \pm}$vanishing identically in the lower half-plane is an immediate consequence of Lemma 4 (in which we replace $r$ by $y \geq 0$ and assume periodicity $\pi$ ).

3.5.2. Proof of Theorem 11. Case $a \neq 0$. When $a \neq 0$ the conditions (3.20)-(3.21) imply

$$
c=s a, 1-s b=t a, s, t \in \mathbb{R} \backslash\{0\} .
$$

This requires $\operatorname{Im} a \neq 0$ since otherwise $L$ would be real and could not be hypoelliptic in $\mathbb{R}^{2} \backslash 0$. The change of variables $y \rightarrow s y$ yields the expression

$$
L=(a x+(1-t a) y) \partial_{x}+(a x+y) \partial_{y} .
$$

From (3.23) we derive

$$
(a x+y)^{-1} L=\partial_{y}+\frac{(a x+(1-t a) y)(\bar{a} x+y)}{|a x+y|^{2}} \partial_{x} .
$$

Let $\varphi \in \mathcal{C}_{\mathrm{c}}^{\infty}\left(\mathbb{R}^{2}\right)$ be arbitrary. Here and in the sequel we denote by $(\cdot, \cdot)$ and $\|\cdot\|$ the Hermitian product and the norm in $L^{2}\left(\mathbb{R}^{2}\right)$. First we look at

$$
\begin{aligned}
& \operatorname{Re}\left((a x+y)^{-1} L \varphi, i y^{4} \varphi_{x}\right)=\operatorname{Im}\left(\phi_{y}, y^{4} \varphi_{x}\right) \\
+ & \operatorname{Im} \int \frac{(a x+(1-t a) y)(\bar{a} x+y)}{|a x+y|^{2}} y^{4}\left|\varphi_{x}\right|^{2} d x d y .
\end{aligned}
$$

We have

$$
\operatorname{Im}(a x+(1-t a) y)(\bar{a} x+y)=-(\operatorname{Im} a) t y^{2}
$$


and

$$
\begin{gathered}
\operatorname{Im}\left(y^{4} \varphi_{y}, \varphi_{x}\right)=\frac{1}{2 i}\left[\left(\varphi_{x}, y^{4} \varphi_{y}\right)-\left(y^{4} \varphi_{y}, \varphi_{x}\right)\right] \\
=-2 i\left(y^{3} \varphi_{x}, \varphi\right)=2 \operatorname{Im}\left(y^{3} \varphi_{x}, \varphi\right)
\end{gathered}
$$

whence

$$
\begin{gathered}
\operatorname{Re}\left((a x+y)^{-1} L \varphi, i y^{4} \varphi_{x}\right)=2 \operatorname{Im}\left(y^{3} \varphi_{x}, \varphi\right) \\
-t(\operatorname{Im} a) \int|a x+y|^{-2}\left|y^{3} \varphi_{x}\right|^{2} d x d y .
\end{gathered}
$$

Next we look at

$$
\begin{aligned}
& 2 \operatorname{Re}\left((a x+y)^{-1} L \varphi, y \varphi\right)=2 \operatorname{Re}\left(\varphi_{y}, y \varphi\right) \\
+ & 2 \operatorname{Re} \int \frac{(a x+(1-t a) y)(\bar{a} x+y)}{|a x+y|^{2}} y \varphi_{x} \bar{\varphi} d x d y .
\end{aligned}
$$

On the one hand we have

$$
2 \operatorname{Re}\left(\varphi_{y}, y \varphi\right)=-\|\varphi\|^{2}
$$

On the other hand,

$$
\begin{gathered}
2 \operatorname{Re} \int \frac{(a x+(1-t a) y)(\bar{a} x+y)}{|a x+y|^{2}} y \varphi_{x} \bar{\varphi} d x d y \\
=-\int y|\varphi|^{2} \frac{\partial}{\partial x}\left(\operatorname{Re} \frac{(a x+(1-t a) y)(\bar{a} x+y)}{|a x+y|^{2}}\right) d x d y \\
-2 \int y \operatorname{Im}\left(\frac{(a x+(1-t a) y)(\bar{a} x+y)}{|a x+y|^{2}}\right) \operatorname{Im}\left(\varphi_{x} \bar{\varphi}\right) d x d y .
\end{gathered}
$$

In dealing with the first term on the right-hand side we make use of the fact that

$$
\frac{\partial}{\partial x} \operatorname{Re} \frac{(a x+(1-t a) y)(\bar{a} x+y)}{|a x+y|^{2}}=\frac{y P(x, y)}{|a x+y|^{2}},
$$

where, in the notation $a^{\prime}=\operatorname{Re} a, a^{\prime \prime}=\operatorname{Im} a$,

$$
\begin{aligned}
P(x, y)= & a^{\prime} x^{2}\left(t\left(a^{\prime}\right)^{3}+t a^{\prime}\left(a^{\prime \prime}\right)^{2}+2\left(a^{\prime \prime}\right)^{2}\right) \\
& +2 x y\left(t\left(a^{\prime}\right)^{3}+\left(a^{\prime}\right)^{2}+2\left(a^{\prime \prime}\right)^{2}\right)-2 y^{2}\left(t\left(a^{\prime \prime}\right)^{2}-a^{\prime}\right) .
\end{aligned}
$$

In dealing with the second term on the right-hand side we make use again of (3.24). We obtain

$$
\begin{aligned}
& 2 \operatorname{Re} \int \frac{(a x+(1-t a) y)(\bar{a} x+y)}{|a x+y|^{2}} y \varphi_{x} \bar{\varphi} d x d y \\
& =-\int|y|^{2}|\varphi|^{2} \frac{P(x, y)}{|a x+y|^{2}} d x d y+2 t a^{\prime \prime} \operatorname{Im}\left(y^{3} \varphi_{x}, \varphi\right)
\end{aligned}
$$

and, taking (3.26) into account,

$$
\begin{gathered}
2 \operatorname{Re}\left((a x+y)^{-1} L \varphi, y \varphi\right)=-\|\varphi\|^{2} \\
-\int|y|^{2}|\varphi|^{2} \frac{P(x, y)}{|a x+y|^{2}} d x d y+2 t a^{\prime \prime} \operatorname{Im}\left(y^{3} \varphi_{x}, \varphi\right) .
\end{gathered}
$$


Combining this with (3.25) yields

$$
\begin{aligned}
& \operatorname{Re}\left((a x+y)^{-1} L \varphi, i t a^{\prime \prime} y^{4} \varphi_{x}-2 y \varphi\right)=-\|\varphi\|^{2}+4 t a^{\prime \prime} \operatorname{Im}\left(y^{3} \varphi_{x}, \varphi\right) \\
& -\int|y|^{2}|\varphi|^{2} \frac{P(x, y)}{|a x+y|^{2}} d x d y-t^{2} a^{\prime \prime 2} \int|a x+y|^{-2}\left|y^{3} \varphi_{x}\right|^{2} d x d y .
\end{aligned}
$$

As before, $\Delta_{\varepsilon}=\left\{(x, y) \in \mathbb{R}^{2} ; x^{2}+y^{2}<\varepsilon\right\}, \varepsilon>0$; (3.27) implies, for some $C>0$ and all $\varphi \in \mathcal{C}_{\mathrm{c}}^{\infty}\left(\Delta_{\varepsilon}\right)$,

$$
\int|y|^{2}|\varphi|^{2} \frac{|P(x, y)|}{|a x+y|^{2}} d x d y \leq C \varepsilon^{2}\|\varphi\|^{2} .
$$

Given any number $B>0$ we can select $\varepsilon$ sufficiently small that

$$
\int|a x+y|^{-2}\left|y^{3} \varphi_{x}\right|^{2} d x d y \geq B\left\|y^{3} \varphi_{x}\right\|^{2}
$$

for all $\varphi \in \mathcal{C}_{\mathrm{c}}^{\infty}\left(\Delta_{\varepsilon}\right)$. Keep in mind that $a^{\prime \prime}=\operatorname{Im} a \neq 0$; by applying the CauchySchwarz inequality we derive from (3.28):

$$
\begin{aligned}
(1-C \varepsilon)\|\varphi\|^{2}+B t^{2} a^{\prime \prime 2}\left\|y^{3} \varphi_{x}\right\|^{2}-4 t a^{\prime \prime} \operatorname{Im}\left(y^{3} \varphi_{x}, \varphi\right) \\
\quad \leq-\operatorname{Re}\left((a x+y)^{-1} L \varphi, i t a^{\prime \prime} y^{4} \varphi_{x}-2 y \varphi\right) \\
\quad=-\operatorname{Re}\left(L \varphi, i t a^{\prime \prime} \frac{y^{4}}{a x+y} \varphi_{x}-2 \frac{y}{a x+y} \varphi\right) \\
\quad \leq\|L \varphi\|\left(\|\varphi\|^{2}+\left\|y^{3} \varphi_{x}\right\|^{2}\right) .
\end{aligned}
$$

We can select $B$ sufficiently large and $\varepsilon$ sufficiently small that

$$
\begin{aligned}
\frac{1}{2}\left(\|\varphi\|^{2}+\left\|y^{3} \varphi_{x}\right\|^{2}\right) & \leq(1-C \varepsilon)\|\varphi\|^{2}+B t^{2} a^{\prime \prime 2}\left\|y^{3} \varphi_{x}\right\|^{2} \\
& -4 t a^{\prime \prime} \operatorname{Im}\left(y^{3} \varphi_{x}, \varphi\right) .
\end{aligned}
$$

We reach the conclusion that, for a suitably large constant $C^{\prime}>0$ and all $\varphi \in$ $\mathcal{C}_{\mathrm{c}}^{\infty}\left(\Delta_{\varepsilon}\right)$

$$
\|\varphi\|^{2}+\left\|y^{3} \varphi_{x}\right\|^{2} \leq C^{\prime}\|L \varphi\|^{2} .
$$

A consequence of (3.29) is that the map $L \mathcal{C}_{\mathrm{c}}^{\infty}\left(\Delta_{\varepsilon}\right) \ni L \varphi \rightarrow \varphi$ extends as a bounded linear operator $E$ on $L^{2}\left(\Delta_{\varepsilon}\right)$ [extend $E$ by continuity to the closure of $L \mathcal{C}_{\mathrm{c}}^{\infty}\left(\Delta_{\varepsilon}\right)$ in $L^{2}\left(\Delta_{\varepsilon}\right)$ and set $E=0$ on the orthogonal of $L \mathcal{C}_{\mathrm{c}}^{\infty}\left(\Delta_{\varepsilon}\right)$ in $\left.L^{2}\left(\Delta_{\varepsilon}\right)\right]$. We have $E L \varphi=\varphi$ for all $\varphi \in \mathcal{C}_{\mathrm{c}}^{\infty}\left(\Delta_{\varepsilon}\right)$. Now let $E^{\top}: L^{2}\left(\Delta_{\varepsilon}\right) \longrightarrow L^{2}\left(\Delta_{\varepsilon}\right)$ be the transpose of $E$ and let $f \in L^{2}\left(\Delta_{\varepsilon}\right)$ be arbitrary. The distribution $g=E^{\top} f \in L^{2}\left(\Delta_{\varepsilon}\right)$ verifies $\left\langle L^{\top} g, \varphi\right\rangle=\langle f, \varphi\rangle$ for all $\varphi \in \mathcal{C}_{\mathrm{c}}^{\infty}\left(\Delta_{\varepsilon}\right)$.

If we wish to solve the equation $L u=F$ for arbitrary $F \in \mathcal{C}_{\mathrm{c}}^{\infty}\left(\Delta_{\varepsilon}\right)$ it suffices, thanks to Lemma 8 , to solve this equation for $F=y^{2} G, G \in \mathcal{C}_{\mathrm{c}}^{\infty}\left(\Delta_{\varepsilon}\right)$. Since (OUT-E) holds we have $|A(\theta)| \geq c_{\circ}$ for some $c_{\circ}>0$ and all $\theta \in \mathbb{R}$. It is therefore permitted to select, in the preceding argument, $f=-r^{-2} A(\theta)^{-1} F=$ $-r^{-2} A(\theta)^{-1} y^{2} G$; clearly, $f \in L^{2}\left(\Delta_{\varepsilon}\right)$. We have, in the distribution sense,

$$
\begin{aligned}
L\left[r^{2} A(\theta) g\right] & =r^{2} A(\theta) L g+(\operatorname{div} L) r^{2} A(\theta) g \\
& =-r^{2} A(\theta) L^{\top} g=-r^{2} A(\theta) f=F .
\end{aligned}
$$

The proof of Theorem 11 is complete. 
3.6. End of the proof of sufficiency in the class (NO-E). The core of Theorems 5(i), 9, 10 and 11 can be restated as follows:

Theorem 12. Let the vector field (1.1) satisfy (NO-E) and be such that $\mu(L) \neq 0$. If $L$ is locally solvable in $\mathbb{R}^{2} \backslash\{0\}$, then it is locally solvable in $\mathbb{R}^{2}$.

Proof. According to Corollary 4, under the hypotheses of Theorem 12, to say that $L$ is hypoelliptic in $\mathbb{R}^{2} \backslash\{0\}$ is the same as saying that $L$ is locally solvable in $\mathbb{R}^{2} \backslash\{0\}$.

\section{Solvability in the Class (ON-E)}

This section is devoted to the proof of the following statement:

Theorem 13. If the vector field (1.1) satisfies Condition $(\boldsymbol{O N}-\mathbf{E})$, then the following conditions are equivalent:

(a): $L$ is locally solvable in $\mathbb{R}^{2} \backslash\{0\}$;

(b): $L$ is locally solvable in $\mathbb{R}^{2}$.

Theorems 12 and 13 combined are equivalent to the proof of the sufficiency of the condition in Theorems 1 . 3 .

4.1. Preparatory reductions. Condition (ON-E) means that $A\left(\theta^{\circ}\right)=0$ for some $\theta^{\circ}$. After a rotation we may assume that $\theta^{\circ}=0$, implying $c=0$ [see (1.3), $\alpha=a-d]$ :

$$
\begin{gathered}
A(\theta)=(b \sin \theta+\alpha \cos \theta) \sin \theta, \\
A^{\prime}(\theta)=b \sin 2 \theta+\alpha \cos 2 \theta .
\end{gathered}
$$

If $c=0$, then

$$
\operatorname{Im}\left(\left(a+d+A^{\prime}(\theta)\right) \overline{A(\theta)}\right)=-2 \operatorname{Im}(\bar{d} A(\theta)) .
$$

Proof. The formulas (4.1) imply

$$
\begin{gathered}
\operatorname{Im}((\alpha+2 d+b \sin 2 \theta+\alpha \cos 2 \theta)(\bar{b} \sin \theta+\bar{\alpha} \cos \theta)) \\
=-2(\operatorname{Im}(b \bar{d}) \sin \theta+\operatorname{Im}(\alpha \bar{d}) \cos \theta) .
\end{gathered}
$$

Proposition 11. Let $L$ be the vector field (1.1). If $c=0$ and if $\operatorname{Im}(\bar{d} A(\theta))$ vanishes identically, then either $d=0$ or $L$ is proportional to a real vector field.

Proof. If $d \neq 0$, for $\operatorname{Im}(\bar{d} A(\theta))$ to vanish identically it is necessary that $a=s d$, $b=t d, s, t \in \mathbb{R}$, in which case $d^{-1} L=(s x+t y) \partial_{x}+y \partial_{y}$.

Proposition 12. Let $L$ be the vector field (1.1). If $c=d=0$ (while $|a|+|b| \neq 0)$, then $L$ is locally solvable at the origin.

Proof. If $\operatorname{Im}(a \bar{b})=0$, then $L$ is proportional to a real vector field and the claim is a consequence of Theorem 4. If $\operatorname{Im}(a \bar{b}) \neq 0$ and if $F \in \mathcal{C}_{c}^{\infty}\left(\mathbb{R}^{2}\right)$, then the function

$$
u(x, y)=\int_{0}^{x} \frac{F(s, y)-F(0, y)}{a s+b y} d s+a^{-1} F(0, y) \log |x|
$$

is a distribution solution of $L u=F$ in $\mathbb{R}^{2}$. 
In the remainder of the proof of Theorem 13 we assume that $c=0$ and that $\operatorname{Im}(\bar{d} A(\theta))$ does not vanish identically, in particular $d \neq 0$. After division of $L$ by $d$ this amounts to saying that $\operatorname{Im} A(\theta)$ does not vanish identically. The choice $c=0, d=1$ means that we are dealing with a vector field

$$
L=(a x+b y) \partial_{x}+y \partial_{y} .
$$

We can exclude the case $a=0$, equivalent to $L=y\left(b \partial_{x}+\partial_{y}\right)$, and obviously solvable. We underline the important consequence

$$
\operatorname{det} \mathfrak{m}(L)=a \neq 0 .
$$

Proposition 13. Let $L$ be the vector field (4.3) with $a \neq 0$. Suppose that $\operatorname{Im} A$ does not vanish identically and that there is $\theta^{*} \in(0, \pi)$ such that

$$
\operatorname{Re}\left(\left(a+1+A^{\prime}\left(\theta^{*}\right)\right) \overline{A\left(\theta^{*}\right)}\right) \neq 0, \operatorname{Im} A\left(\theta^{*}\right)=0 .
$$

In this case $L$ is not locally solvable at any point of the straight line $\theta=\theta^{*}$.

Proof. In a conic neighborhood of the ray $\left(r \cos \theta^{*}, r \sin \theta^{*}\right)$ we have $A(\theta) \neq 0$ and

$$
\omega^{\prime}(\theta)=\frac{1}{2} \operatorname{Im}\left(\frac{a+1+A^{\prime}(\theta)}{A(\theta)}\right)=-2 \frac{\operatorname{Im} A(\theta)}{|A(\theta)|^{2}}
$$

is well-defined and vanishes at $\theta^{*}$. We also have

$$
\partial_{\theta}\left(\frac{\operatorname{Im} A(\theta)}{|A(\theta)|^{2}}\right)=\frac{\operatorname{Im} A^{\prime}(\theta)}{|A(\theta)|^{2}}-2 \frac{(\operatorname{Im} A(\theta))}{|A(\theta)|^{4}} \operatorname{Re}\left(A^{\prime}(\theta) \overline{A(\theta)}\right) .
$$

Suppose we had $\operatorname{Im} A^{\prime}\left(\theta^{*}\right)=0$; we would have simultaneously

$$
\begin{aligned}
(\operatorname{Im} b) \sin ^{2} \theta^{*}+(\operatorname{Im} a) \cos \theta^{*} \sin \theta^{*} & =0 \\
(\operatorname{Im} b) \sin 2 \theta^{*}+(\operatorname{Im} a) \cos 2 \theta^{*} & =0,
\end{aligned}
$$

requiring either $\operatorname{Im} a=\operatorname{Im} b=0$, in which case $\operatorname{Im} A \equiv 0$, which is excluded, or else

$$
\operatorname{det}\left(\begin{array}{cc}
\sin ^{2} \theta^{*} & \frac{1}{2} \sin 2 \theta^{*} \\
\sin 2 \theta^{*} & \cos 2 \theta^{*}
\end{array}\right)=-\sin ^{2} \theta^{*}=0,
$$

contradicting the hypothesis that $\theta^{*} \in(0, \pi)$. We conclude that $\omega^{\prime \prime}\left(\theta^{*}\right) \neq 0$ and, therefore, that $\omega^{\prime}$ changes sign at $\theta^{*}$, whence the claim by Proposition 4 .

We can restate Proposition 13 as follows:

If $L$ is locally solvable in $\mathbb{R}^{2} \backslash\{0\}$ (with $c=0, d=1$ ) and if $\operatorname{Im} A(\theta)$ does not vanish identically, then

$$
\forall \theta \in \mathbb{R}, \operatorname{Im} A(\theta)=0 \Longrightarrow \operatorname{Re}\left(\left(a+1+A^{\prime}(\theta)\right) \overline{A(\theta)}\right)=0 .
$$

Proposition 14. If $L$ is given by (4.3) there is a linear change of coordinates $x, y$ achieving $(\operatorname{Im} a)(\operatorname{Im} b)=0$.

Proof. We use the notation $a^{\prime}=\operatorname{Re} a, a^{\prime \prime}=\operatorname{Im} a, b^{\prime}=\operatorname{Re} b, b^{\prime \prime}=\operatorname{Im} b$. If $a^{\prime \prime} b^{\prime \prime} \neq 0$ we see that

$$
\begin{gathered}
\left(\begin{array}{cc}
1 & \frac{1}{a^{\prime \prime}} b^{\prime \prime} \\
0 & -\frac{1}{a^{\prime \prime}} b^{\prime \prime}
\end{array}\right)\left(\begin{array}{cc}
a^{\prime} & b^{\prime} \\
0 & 1
\end{array}\right)\left(\begin{array}{cc}
1 & 1 \\
0 & -\frac{a^{\prime \prime}}{b^{\prime \prime}}
\end{array}\right)=\left(\begin{array}{cc}
a^{\prime} & a^{\prime}-1-\frac{a^{\prime \prime}}{b^{\prime \prime}} b^{\prime} \\
0 & 1
\end{array}\right), \\
\left(\begin{array}{cc}
1 & \frac{1}{a^{\prime \prime}} b^{\prime \prime} \\
0 & -\frac{1}{a^{\prime \prime}} b^{\prime \prime}
\end{array}\right)\left(\begin{array}{cc}
a^{\prime \prime} & b^{\prime \prime} \\
0 & 0
\end{array}\right)\left(\begin{array}{cc}
1 & 1 \\
0 & -\frac{a^{\prime \prime}}{b^{\prime \prime}}
\end{array}\right)=\left(\begin{array}{cc}
a^{\prime \prime} & 0 \\
0 & 0
\end{array}\right) .
\end{gathered}
$$


This shows that a linear change of variables brings us to the case where $b$ is real. We conclude that we can always assume either $a \in \mathbb{R}, b^{\prime \prime} \neq 0$, or else $a^{\prime \prime} \neq 0$, $b \in \mathbb{R}$.

Proposition 15. Suppose $c=0, d=1$ and $b \in \mathbb{R}$. If $L$ is locally solvable in $\mathbb{R}^{2} \backslash\{0\}$, then necessarily $b=0$.

Proof. When $b \in \mathbb{R}$ we derive from (4.1) and (4.2), on the one hand,

$$
\operatorname{Im}\left(\left(a+1+A^{\prime}(\theta)\right) \overline{A(\theta)}\right)=-(\operatorname{Im} \alpha) \sin 2 \theta,
$$

which vanishes at $\frac{\pi}{2}$; and on the other hand,

$$
A\left(\frac{\pi}{2}\right)=b, A^{\prime}\left(\frac{\pi}{2}\right)=-(a-1)
$$

whence

$$
\operatorname{Re}\left(\left(a+1+A^{\prime}\left(\frac{\pi}{2}\right)\right) \overline{A\left(\frac{\pi}{2}\right)}\right)=-2 b,
$$

which must also vanish, by (4.5).

We are left with the following two cases to settle:

Case I: $\operatorname{Im} b \neq 0, a \in \mathbb{R} \backslash\{0\}$. In this case we are dealing with the vector field

$$
L=\left(a x+b^{\prime} y+i b^{\prime \prime} y\right) \partial_{x}+y \partial_{y}, 0 \neq a \in \mathbb{R} .
$$

We carry out the change of variable $y \rightarrow b^{\prime \prime-1} y$ and a redefinition of $b^{\prime}$, thus getting

$$
L=\left(a x+b^{\prime} y+i y\right) \partial_{x}+y \partial_{y}, 0 \neq a \in \mathbb{R} .
$$

Case II: $b=0, \operatorname{Im} a \neq 0$ (otherwise $L$ is real). In this case

$$
A(\theta)=(a-1) \cos \theta \sin \theta
$$

vanishes for $\theta=0, \theta=\frac{\pi}{2}$.

The techniques to handle these two cases will be different.

4.1.1. Global solvability in the case $\operatorname{Im} b \neq 0$. We shall prove that, in this case, the equation $L u=F, F \in \mathcal{C}_{\mathrm{c}}^{\infty}\left(\mathbb{R}^{2}\right)$, is solvable in $L^{2}\left(\mathbb{R}^{2}\right)$.

As before we denote by $(\cdot, \cdot)$ and $\|\cdot\|$ the Hermitian product and the norm in $L^{2}\left(\mathbb{R}^{2}\right)$.

Lemma 9. Let $L$ be as in (4.6). Given any $\lambda \in \mathbb{R}$ and any $\varphi \in \mathcal{C}_{c}^{\infty}\left(\mathbb{R}^{2}\right)$, we have

$$
\operatorname{Re}\left(L \varphi, i \lambda y \varphi_{x}-\varphi\right)=\lambda\left\|y \varphi_{x}\right\|^{2}+(\lambda+1) \operatorname{Im}\left(y \varphi_{x}, \varphi\right)+(1+a)\|\varphi\|^{2} .
$$

Proof. On the one hand,

$$
\begin{gathered}
\operatorname{Re}\left(L \varphi, i \lambda y \varphi_{x}-\varphi\right)=\operatorname{Re}\left(\left(a x+b^{\prime} y\right) \varphi_{x}, i \lambda y \varphi_{x}\right)+\lambda\left\|y \varphi_{x}\right\|^{2}+\lambda \operatorname{Re}\left(y \varphi_{y}, i y \varphi_{x}\right) \\
-a \operatorname{Re}\left(x \partial_{x} \varphi, \varphi\right)-\operatorname{Re}\left(y \partial_{y} \varphi, \varphi\right)-b^{\prime} \operatorname{Re}\left(y \varphi_{x}, \varphi\right)-\operatorname{Re}\left(i y \varphi_{x}, \varphi\right) \\
=\lambda\left\|y \varphi_{x}\right\|^{2}+(1+a)\|\varphi\|^{2}+\lambda \operatorname{Im}\left(y^{2} \varphi_{y}, \varphi_{x}\right)+\operatorname{Im}\left(y \varphi_{x}, \varphi\right) .
\end{gathered}
$$

On the other hand,

$$
\begin{aligned}
\left(y^{2} \varphi_{y}, \varphi_{x}\right)-\left(\varphi_{x}, y^{2} \varphi_{y}\right) & =\left(\partial_{y}\left(y^{2} \varphi_{x}\right), \varphi\right)-\left(\varphi, \partial_{y}\left(y^{2} \varphi_{x}\right)\right) \\
& =2\left[\left(y \varphi_{x}, \varphi\right)-\left(\varphi, y \varphi_{x}\right)\right]+\left(y^{2} \varphi_{x y}, \varphi\right)-\left(\varphi, y^{2} \varphi_{x y}\right) \\
& =2\left[\left(y \varphi_{x}, \varphi\right)-\left(\varphi, y \varphi_{x}\right)\right]-\left(y^{2} \varphi_{y}, \varphi_{x}\right)+\left(\varphi_{x}, y^{2} \varphi_{y}\right)
\end{aligned}
$$


whence

$$
\frac{1}{2 i}\left[\left(y^{2} \varphi_{y}, \varphi_{x}\right)-\left(\varphi_{x}, y^{2} \varphi_{y}\right)\right]=\frac{1}{2 i}\left[\left(y \varphi_{x}, \varphi\right)-\left(\varphi, y \varphi_{x}\right)\right] .
$$

This directly implies (4.7).

Corollary 8. Let $L$ be as in (4.6). For all $\lambda \in \mathbb{R}$ and $\varphi \in \mathcal{C}_{c}^{\infty}\left(\mathbb{R}^{2}\right)$ we have

$$
\operatorname{Re}\left((L+1) \varphi, i \lambda y \varphi_{x}-\varphi\right)=\lambda\left\|y \varphi_{x}\right\|^{2}+\operatorname{Im}\left(y \varphi_{x}, \varphi\right)+a\|\varphi\|^{2} .
$$

Proof. Indeed,

$$
\operatorname{Re}\left(\varphi, i \lambda y \varphi_{x}-\varphi\right)=\operatorname{Re}\left(\varphi, i y \varphi_{x}\right)-\|\varphi\|^{2}=-\operatorname{Im}\left(y \varphi_{x}, \varphi\right)-\|\varphi\|^{2} .
$$

Proposition 16. Let $L$ be as in (4.6). To each $F \in \mathcal{C}_{c}^{\infty}\left(\mathbb{R}^{2}\right)$ there is $u \in L^{2}\left(\mathbb{R}^{2}\right)$ such that $L u=F$.

Proof. In (4.8) we select $\lambda \in \mathbb{R}$ such that $1<4 \lambda a$. We obtain, for some $C>0$ and all $\varphi \in \mathcal{C}_{\mathrm{c}}^{\infty}\left(\mathbb{R}^{2}\right)$,

$$
\left\|y \varphi_{x}\right\|+\|\varphi\| \leq C\|(L+1) \varphi\| .
$$

Since $(L+1)^{\top}=-(L+a)$ the estimate (4.9) implies the existence of $v \in L^{2}\left(\mathbb{R}^{2}\right)$ such that $y \partial_{x} v \in L^{2}\left(\mathbb{R}^{2}\right)$, verifying

$$
L v+a v=F_{x} .
$$

Then define $\phi(x, y)=\int_{0}^{x} v(s, y) d s$; we get

$$
\begin{aligned}
L \phi & =(a x+b y) v(x, y)+\int_{0}^{x} y \partial_{y} v(s, y) d s \\
& =(a x+b y) v(x, y)+\int_{0}^{x} L v(s, y) d s-\int_{0}^{x}(a s+b y) \partial_{s} v(s, y) d s \\
& =(a x+b y) v(x, y)+F(x, y)-F(0, y)-\left.(a s+b y) v(s, y)\right|_{s=0} ^{s=x} \\
& =F(x, y)-F(0, y)+b y v(0, y) .
\end{aligned}
$$

Note in passing that $y v(0, y)$ is well-defined since $\partial_{x}(y v) \in L^{2}$. If we select

$$
u(x, y)=\phi(x, y)+F(0,0) \log |y|+\int_{0}^{y} \frac{F(0, t)-F(0,0)}{t} d t-b \int_{0}^{y} v(0, t) d t,
$$

it is readily checked that $L u=F$ and $u \in L^{2}\left(\mathbb{R}^{2}\right)$.

4.1.2. Local solvability in the case $b=0$. Here we have

$$
L=a x \partial_{x}+y \partial_{y}, \operatorname{Im} a \neq 0 .
$$

A first integral of $L$ is

$$
Z(x, y)=a^{-1} \log |x|-\log |y| .
$$

For use below we note that

$$
d Z \wedge d \bar{Z}=2 i\left(\operatorname{Im}\left(a^{-1}\right)\right) x^{-1} y^{-1} d x \wedge d y .
$$

Lemma 10. If $L$ is the vector field (4.11), then to each $F \in \mathcal{C}^{\infty}\left(\mathbb{R}^{2}\right)$ there is $\varphi \in L_{\text {loc }}^{1}\left(\mathbb{R}^{2}\right)$ such that $L \varphi-F$ belongs to $\mathcal{C}^{\infty}\left(\mathbb{R}^{2}\right)$ and is divisible by $x y$. 
Proof. First take

$$
\phi(y)=F(0,0) \log |y|+\int_{0}^{y}(F(0, t)-F(0,0)) \frac{d t}{t} .
$$

We get $L[\phi(y)]=y \phi^{\prime}(y)=F(0, y)$. Then take

$$
\psi(x, y)=a^{-1} \int_{0}^{x}[F(s, y)-F(0, y)] \frac{d s}{s} .
$$

We get

$$
L \psi=F(x, y)-F(0, y)+a^{-1} y \int_{0}^{x}\left[\partial_{y} F(s, y)-\partial_{y} F(0, y)\right] \frac{d s}{s} .
$$

We conclude that

$$
L[\phi(y)+\psi(x, y)]-F(x, y)=a^{-1} y \int_{0}^{x}\left[\partial_{y} F(s, y)-\partial_{y} F(0, y)\right] \frac{d s}{s} .
$$

Proposition 17. Let $L$ be the vector field (4.11) and let $\Omega \subset \mathbb{R}^{2}$ be a bounded open set containing the origin. To every $F \in \mathcal{C}_{c}^{\infty}(\Omega)$ there is $u \in L_{\text {loc }}^{1}\left(\mathbb{R}^{2}\right)$ such that $L u=F$ in $\Omega$.

Proof. According to Lemma 10 there is a function $\varphi \in L_{\text {loc }}^{1}\left(\mathbb{R}^{2}\right)$ such that $L \varphi-F=$ $x y G$ with $G \in \mathcal{C}^{\infty}\left(\mathbb{R}^{2}\right)$. Let $\chi \in \mathcal{C}_{\mathrm{c}}^{\infty}\left(\mathbb{R}^{2}\right), \chi(x, y)=1$ for all $(x, y) \in \Omega$. To prove the claim it suffices to solve the equation $L v=x y \chi G$ in $\mathbb{R}^{2}$. Actually we are going to construct a solution $v_{++} \in \mathcal{C}^{0}\left(\mathbb{R}^{2}\right)$ of the equation $L v_{++}=\Psi_{++}$in $\mathbb{R}^{2}$, where $\Psi_{++}(x, y)=H(x) H(y) x y \chi G(x, y) ; v_{++}(x, y)$ will vanish in the complement of the quadrant $x>0, y>0$. It will be evident that the same method applies in the other quadrants, yielding continuous solutions $v_{+-}, v_{-+}$and $v_{--}$, each vanishing identically in the complements of their respective quadrants. It will then suffice to take $v=v_{++}+v_{+-}+v_{-+}+v_{--}$. In the notation (4.12) and taking (4.13) into account we define, for $x>0, y>0$,

$$
v_{++}(x, y)=\frac{1}{2 i \pi} \frac{\operatorname{Im} a}{|\operatorname{Im} a|} a^{-1} \iint \frac{(\chi G)(\xi, \eta)}{Z(x, y)-Z(\xi, \eta)} d \xi d \eta .
$$

We carry out the change of variables $\xi=e^{s}, \eta=e^{t},(s, t) \in \mathbb{R}^{2}$ and set $(\chi G)(\xi, \eta)=$ $(\chi G)^{*}(s, t) \in \mathcal{C}^{\infty}\left(\mathbb{R}^{2}\right) \cap L^{\infty}\left(\mathbb{R}^{2}\right)$; we get

$$
v_{++}(x, y)=\frac{1}{2 i \pi} \frac{\operatorname{Im} a}{|\operatorname{Im} a|} a^{-1} \iint_{\mathbb{R}^{2}} \frac{(\chi G)^{*}(s, t)}{Z(x, y)-\left(a^{-1} s-t\right)} e^{s+t} d s d t .
$$

The domain of integration in (4.14) can be restricted to the region

$$
\mathfrak{R}=\left\{(s, t) \in \mathbb{R}^{2} ;\left(e^{s}, e^{t}\right) \in \operatorname{supp} \chi\right\} .
$$

We carry out the change of variables $x=e^{X}, y=e^{Y}$, which transforms $L$ into $\widetilde{L}=a \frac{\partial}{\partial X}+\frac{\partial}{\partial Y}$. We get

$$
v_{++}^{*}(X, Y)=v_{++}\left(e^{X}, e^{Y}\right)=\frac{1}{2 i \pi} \frac{\operatorname{Im} a}{|\operatorname{Im} a|} \iint \frac{(\chi G)^{*}(s, t) e^{s+t}}{X-a Y-(s-a t)} d s d t .
$$

We point out that $(\chi G)^{*}(s, t) e^{s+t}$ tends to zero exponentially fast at infinity: it vanishes identically in the region $\max (s, t)>R$ for some $R>0$ and $(\chi G)^{*}$ is uniformly bounded in $\mathbb{R}^{2}$. To make matters simpler we carry out the linear changes 
of variables $X-(\operatorname{Re} a) Y \rightarrow X, Y \rightarrow-(\operatorname{Im} a) Y$ and $s-(\operatorname{Re} a) t \rightarrow s, t \rightarrow-(\operatorname{Im} a) t$, yielding

$$
v_{++}^{*}(X, Y)=v_{++}\left(e^{X}, e^{Y}\right)=\frac{1}{2 i \pi \operatorname{Im} a} \iint \frac{g(s, t)}{X+i Y-(s+i t)} d s d t .
$$

In the new coordinates, $L=i(\operatorname{Im} a)\left(\frac{\partial}{\partial X}+i \frac{\partial}{\partial Y}\right)$ whose fundamental solution is $\frac{1}{2 i \pi(\operatorname{Im} a)} \frac{1}{X+i Y}$, whence

$$
\widetilde{L} v_{++}^{*}(X, Y)=g(X, Y) .
$$

Backtracking one can check that $L v_{++}=x y \chi G$. Since $(X+i Y)^{-1} \in L_{\text {loc }}^{1}\left(\mathbb{R}^{2}\right)$ the standard estimate for convolution yields $v_{++} \in L^{\infty}\left(\mathbb{R}^{2}\right)$. The ellipticity of $\frac{\partial}{\partial \bar{Z}}$ implies $v_{++}^{*} \in \mathcal{C}^{\infty}\left(\mathbb{R}^{2}\right)$ and, therefore, that $v_{++}$is smooth in the quadrant $x>0$, $y>0$. The Lebesgue dominated convergence theorem implies that if $X \rightarrow-\infty$ or $Y \rightarrow-\infty$, then $v_{++}^{*}(X, Y)$ defined by (4.15) tends to zero. This shows that if $x y \rightarrow 0$, then $v_{++}(x, y) \rightarrow 0$, proving that $v_{++}$can be extended as a continuous function in $\mathbb{R}^{2}$, identically zero in the complement of the quadrant $x>0, y>0$, as desired.

This completes the proof of Theorem 13 .

\section{A MICROLOCAL REMARK}

5.1. Property (NO-E) in phase space. We recall that the symbol of the vector field (1.1) is the following function in the phase space $\mathbb{R}_{x, y}^{2} \times \mathbb{R}_{\xi, \eta}^{2}$ :

$$
\sigma(L)(x, y, \xi, \eta)=(a x+b y) \xi+(c x+d y) \eta .
$$

The Hamiltonian vector field of $\sigma(L)$ is the vector field $H_{L}=L-L^{\prime}$ in $\mathbb{R}_{x, y}^{2} \times \mathbb{R}_{\xi, \eta}^{2}$, where

$$
L^{\prime}=(a \xi+c \eta) \frac{\partial}{\partial \xi}+(b \xi+d \eta) \frac{\partial}{\partial \eta} .
$$

Thus, $\mathfrak{m}\left(L^{\prime}\right)=\mathfrak{m}(L)^{\top}$, the transpose of $\mathfrak{m}(L)$. We also introduce the "radial" vector field in the "fibre directions", $\xi \frac{\partial}{\partial \xi}+\eta \frac{\partial}{\partial \eta}$. We recall the following terminology from the general theory of linear PDEs. The vector field $L$ is said to be of principal type at a point $\wp \in \mathbb{R}_{x, y}^{2} \times\left(\mathbb{R}_{\xi, \eta}^{2} \backslash\{0\}\right)$ if $H_{L}$ and $\left(\xi \frac{\partial}{\partial \xi}+\eta \frac{\partial}{\partial \eta}\right)$ are linearly independent at $\wp$. Obviously $L$ is of principal type at any point $(x, y, \xi, \eta) \in \mathbb{R}_{x, y}^{2} \times\left(\mathbb{R}_{\xi, \eta}^{2} \backslash\{0\}\right)$ whose base projection $(x, y)$ is not a critical point of $L$. For $L$ to be of principal type, meaning at every point of $\mathbb{R}_{x, y}^{2} \times\left(\mathbb{R}_{\xi, \eta}^{2} \backslash\{0\}\right)$, it is necessary and sufficient that $H_{L} \wedge\left(\xi \frac{\partial}{\partial \xi}+\eta \frac{\partial}{\partial \eta}\right) \neq 0$ at every point $(x, y, \xi, \eta)$ such that $(x, y)$ is a critical point of $L$ and $(\xi, \eta) \neq(0,0)$. At such points we can replace $H_{L}$ by $L^{\prime}$. We have

$$
\left(\xi \frac{\partial}{\partial \xi}+\eta \frac{\partial}{\partial \eta}\right) \wedge L^{\prime}=\left(b \xi^{2}-(a-d) \xi \eta-c \eta^{2}\right) \frac{\partial}{\partial \xi} \wedge \frac{\partial}{\partial \eta} .
$$

Proposition 18. If the origin is the only critical point of the vector field (1.1), then Property $(\boldsymbol{N O}-\mathbf{E})$ is equivalent to the fact that $L$ is of principal type. 
Proof. If we write $\xi=\rho \cos \omega, \eta=\rho \sin \omega$, comparison of (1.3) and (5.3) shows that

$$
b \xi^{2}-(a-d) \xi \eta-c \eta^{2}=\rho^{2} A\left(\theta-\frac{\pi}{2}\right)
$$

proving that (5.3) does not vanish at any point of $\mathbb{R}_{\xi, \eta}^{2} \backslash\{0\}$ if and only if $A(\theta) \neq 0$ for all $\theta \in \mathbb{R}$.

5.2. Comparing Theorem 12 to a classical theorem of Hörmander. Suppose that the origin is the only critical point of the vector field (1.1) and that $L$ is of principal type or, equivalently, that $L$ satisfies Condition (NO-E). Let us denote by $\mathfrak{g}\left(H_{L}\right)$ the real Lie algebra (for the commutation bracket) generated by the vector fields $\operatorname{Re} H_{L}$ and $\operatorname{Im} H_{L}$. The Nagano theorem ([Nagano, 1966] ) states that the whole phase space $\mathbb{R}_{x, y}^{2} \times \mathbb{R}_{\xi, \eta}^{2}$ is foliated by "integral manifolds" of $\mathfrak{g}\left(H_{L}\right)$; these are connected, immersed analytic submanifolds of $\mathbb{R}_{x, y}^{2} \times \mathbb{R}_{\xi, \eta}^{2}$ whose tangent space at every point $\wp$ is equal to the freezing of $\mathfrak{g}\left(H_{L}\right)$ at $\wp$ and which are maximal for such properties. These submanifolds will be referred to as the $H_{L}$-leaves.

Since $H_{L}=L_{x, y}-L_{\xi, \eta}^{\prime}$ we see that any $H_{L}$-leaf intersecting the "vertical" plane $\{0\} \times \mathbb{R}_{\xi, \eta}^{2}$ is entirely contained in it. It ensues that there exist $H_{L}$-leaves whose base projection is a single point, $\{0\}$. Theorem 12 states that the vector field (1.1) verifying (NO-E), and therefore of principal type, is locally solvable in $\mathbb{R}^{2}$ provided that it be locally solvable in $\mathbb{R}^{2} \backslash\{0\}$ and not have compact orbits in $\mathbb{R}^{2} \backslash\{0\}$. This result stands in sharp contrast with Theorem 26.11.3 in [Hörmander, 1985], stating, as a sufficient condition for a PDE of principal type $P(x, D)$ to be semiglobally solvable, that $P(x, D)$ satisfy the local solvability condition $(\mathbf{P})$ and that the base projections of every one of its "bicharacteristics" escape from every compact subset of the base, i.e., not be "trapped". This suggests that the conclusion in Theorem 26.11.3 loc.cit., might be valid even when trapped bicharacteristics are present, provided there be at least one that is not trapped. At any rate it raises some question as to the appropriateness of Definition 26.11.4 loc. cit.

\section{REFERENCES}

[Bergamasco-Meziani, 2005] Bergamasco, A. P. and Meziani, A. Solvability near the characteristic set for a class of planar vector fields of infinite type, Ann. Inst. Fourier, Grenoble 55 (2005), 77-112. MR2141289 (2005m:35028)

[Cordaro-Gong, 2004] Cordaro, P. D. and Gong, X. Normalization of complex-valued planar vector fields which degenerate along a real curve, Advances in Math. 184 (2004), 89-118. MR2047850 (2005a:35004)

[Hörmander, 1959] Hörmander, L. On the range of convolution operators, Ann. of Math. (2) 76 (1962), 148-170. MR0141984 (25:5379)

[Hörmander, 1969]

[Hörmander, 1985]

Hörmander, L. Linear Partial Differential Operators, SpringerVerlag, Berlin, 1969. MR0248435 (40:1687)

Hörmander, L. The Analysis of Linear Partial Differential Equations IV, Springer-Verlag, Berlin, 1985. MR0781537 (87d:35002b)

[Lojasiewiccz, 1965]

[Meziani, 2001] Lojasiewicz, S., Notes, Institut Hautes Études, Bures-sur-Yvette, 1965.

Meziani, A. On planar elliptic structures with infinite type degeneracy, J. Funct. Anal. 179 (2001), 333-373. MR1809114 (2001k:35122)

[Meziani, 2004]

Meziani, A. Elliptic vector fields with degeneracies, Trans. Amer. Math. Soc. 357 (2004) 4225-4248. MR2159708 (2006f:35107)

[Miwa, 1973]

Miwa, T. On the existence of hyperfunction solution of Linear Differential Equations of the first order with degenerate real principal symbol, Proc. Japan Acad. 49 (1973), 88-93. MR0348236 (50:734) 
[Müller, 1992]

[Nagano, 1966]

[Nirenberg-Treves, 1963]

[Treves, 1971]

[Treves, 1992]

[Treves, 2009]
Müller, D. H. Local solvability of first order differential operators near a critical point, operators with quadratic symbols and the Heisenberg group, Comm. P. D. E. 17 (1992), 305-337. MR1151265 (93g:35005)

Nagano, T., Linear differential systems with singularities and applications to transitive Lie algebras, J. Math. Soc. Japan 18 (1966), 398-404. MR0199865 (33:8005)

Nirenberg, L. and Treves, F. Solvability of a first-order linear partial differential equation, Comm. Pure Appl. Math. 16 (1963), 331-351. MR0163045 (29:348)

Treves, F., Analytic-Hypoelliptic Partial Differential Equations of Principal Type, Comm. Pure Applied Math. XXIV (1971), 537-570. MR0296509(45:5569)

Treves, F., Hypo-Analytic Structures, Local Theory, Princeton University Press, Princeton, N. J., 1992. MR.1200459 (94e:35014)

Treves, F., On the solvability of vector fields with real linear coefficients, Proceedings Amer. Math. Soc. 137 (2009), 4209-4218. MR 2538582 (2010k:35060)

12002 Spruce Canyon Circle, Golden, Colorado 80403

E-mail address: treves.jeanfrancois@gmail.com 